\title{
Suplementary Material
}

Dipole moments and polarizabilties used to estimate the collision rate of charged clusters.

\begin{tabular}{|c|c|c|}
\hline & Dipole moment (D) & Polarizability $\left(\AA^{3}\right)$ \\
\hline $\mathrm{H}_{2} \mathrm{SO}_{4}$ & $2.96^{\mathrm{a}}$ & $6.2^{\mathrm{b}}$ \\
\hline$\left(\mathrm{H}_{2} \mathrm{SO}_{4}\right)_{2}$ & 0.002 & 9.061 \\
\hline$\left(\mathrm{H}_{2} \mathrm{SO}_{4}\right)_{3}$ & 3.692 & 13.710 \\
\hline$\left(\mathrm{H}_{2} \mathrm{SO}_{4}\right)_{4}$ & 3.252 & 18.759 \\
\hline $\mathrm{NH}_{3}$ & $1.47^{\mathrm{c}}$ & $2.81^{\mathrm{c}}$ \\
\hline $\mathrm{H}_{2} \mathrm{SO}_{4} \cdot \mathrm{NH}_{3}$ & 5.259 & 6.073 \\
\hline$\left(\mathrm{H}_{2} \mathrm{SO}_{4}\right)_{2} \cdot \mathrm{NH}_{3}$ & 9.309 & 10.733 \\
\hline$\left(\mathrm{H}_{2} \mathrm{SO}_{4}\right)_{3} \cdot \mathrm{NH}_{3}$ & 4.580 & 15.482 \\
\hline$\left(\mathrm{H}_{2} \mathrm{SO}_{4}\right)_{4} \cdot \mathrm{NH}_{3}$ & 8.006 & 20.171 \\
\hline$\left(\mathrm{H}_{2} \mathrm{SO}_{4}\right)_{5} \cdot \mathrm{NH}_{3}$ & 4.103 & 24.822 \\
\hline$\left(\mathrm{NH}_{3}\right)_{2}$ & 0.000 & 3.106 \\
\hline $\mathrm{H}_{2} \mathrm{SO}_{4} \cdot\left(\mathrm{NH}_{3}\right)_{2}$ & 3.990 & 7.826 \\
\hline$\left(\mathrm{H}_{2} \mathrm{SO}_{4}\right)_{2} \cdot\left(\mathrm{NH}_{3}\right)_{2}$ & 6.762 & 12.455 \\
\hline$\left(\mathrm{H}_{2} \mathrm{SO}_{4}\right)_{3} \cdot\left(\mathrm{NH}_{3}\right)_{2}$ & 10.065 & 17.121 \\
\hline$\left(\mathrm{H}_{2} \mathrm{SO}_{4}\right)_{4} \cdot\left(\mathrm{NH}_{3}\right)_{2}$ & 9.769 & 21.828 \\
\hline$\left(\mathrm{H}_{2} \mathrm{SO}_{4}\right)_{5} \cdot\left(\mathrm{NH}_{3}\right)_{2}$ & 8.476 & 26.755 \\
\hline$\left(\mathrm{NH}_{3}\right)_{3}$ & 0.000 & 4.964 \\
\hline $\mathrm{H}_{2} \mathrm{SO}_{4} \cdot\left(\mathrm{NH}_{3}\right)_{3}$ & 7.430 & 9.633 \\
\hline$\left(\mathrm{H}_{2} \mathrm{SO}_{4}\right)_{2} \cdot\left(\mathrm{NH}_{3}\right)_{3}$ & 4.006 & 14.184 \\
\hline$\left(\mathrm{H}_{2} \mathrm{SO}_{4}\right)_{3} \cdot\left(\mathrm{NH}_{3}\right)_{3}$ & 7.700 & 18.719 \\
\hline$\left(\mathrm{H}_{2} \mathrm{SO}_{4}\right)_{4} \cdot\left(\mathrm{NH}_{3}\right)_{3}$ & 7.163 & 23.637 \\
\hline$\left(\mathrm{H}_{2} \mathrm{SO}_{4}\right)_{5} \cdot\left(\mathrm{NH}_{3}\right)_{3}$ & 5.099 & 28.112 \\
\hline$\left(\mathrm{NH}_{3}\right)_{4}$ & 0.000 & 6.797 \\
\hline $\mathrm{H}_{2} \mathrm{SO}_{4} \cdot\left(\mathrm{NH}_{3}\right)_{4}$ & 5.798 & 11.458 \\
\hline$\left(\mathrm{H}_{2} \mathrm{SO}_{4}\right)_{2} \cdot\left(\mathrm{NH}_{3}\right)_{4}$ & 4.208 & 15.980 \\
\hline$\left(\mathrm{H}_{2} \mathrm{SO}_{4}\right)_{3} \cdot\left(\mathrm{NH}_{3}\right)_{4}$ & 2.150 & 20.630 \\
\hline$\left(\mathrm{H}_{2} \mathrm{SO}_{4}\right)_{4} \cdot\left(\mathrm{NH}_{3}\right)_{4}$ & 4.011 & 25.383 \\
\hline$\left(\mathrm{H}_{2} \mathrm{SO}_{4}\right)_{5} \cdot\left(\mathrm{NH}_{3}\right)_{4}$ & 8.191 & 30.007 \\
\hline$\left(\mathrm{CH}_{3}\right)_{2} \mathrm{NH}$ & $1.01^{\mathrm{c}}$ & $6.37^{\mathrm{c}}$ \\
\hline $\mathrm{H}_{2} \mathrm{SO}_{4} \cdot\left(\mathrm{CH}_{3}\right)_{2} \mathrm{NH}$ & 8.755 & 9.367 \\
\hline$\left(\mathrm{H}_{2} \mathrm{SO}_{4}\right)_{2} \cdot\left(\mathrm{CH}_{3}\right)_{2} \mathrm{NH}$ & 7.842 & 13.941 \\
\hline$\left(\mathrm{H}_{2} \mathrm{SO}_{4}\right)_{3} \cdot\left(\mathrm{CH}_{3}\right)_{2} \mathrm{NH}$ & 9.113 & 18.640 \\
\hline$\left(\mathrm{H}_{2} \mathrm{SO}_{4}\right)_{4} \cdot\left(\mathrm{CH}_{3}\right)_{2} \mathrm{NH}$ & 7.938 & 23.277 \\
\hline$\left(\left(\mathrm{CH}_{3}\right)_{2} \mathrm{NH}\right)_{2}$ & 0.000 & 10.170 \\
\hline $\mathrm{H}_{2} \mathrm{SO}_{4} \cdot\left(\left(\mathrm{CH}_{3}\right)_{2} \mathrm{NH}\right)_{2}$ & 6.823 & 14.733 \\
\hline$\left(\mathrm{H}_{2} \mathrm{SO}_{4}\right)_{2} \cdot\left(\left(\mathrm{CH}_{3}\right)_{2} \mathrm{NH}\right)_{2}$ & 5.843 & 18.876 \\
\hline$\left(\mathrm{H}_{2} \mathrm{SO}_{4}\right)_{3} \cdot\left(\left(\mathrm{CH}_{3}\right)_{2} \mathrm{NH}\right)_{2}$ & 7.606 & 23.345 \\
\hline$\left(\mathrm{H}_{2} \mathrm{SO}_{4}\right)_{4} \cdot\left(\left(\mathrm{CH}_{3}\right)_{2} \mathrm{NH}\right)_{2}$ & 0.411 & 28.419 \\
\hline$\left(\left(\mathrm{CH}_{3}\right)_{2} \mathrm{NH}\right)_{3}$ & 0.012 & 15.451 \\
\hline $\mathrm{H}_{2} \mathrm{SO}_{4} \cdot\left(\left(\mathrm{CH}_{3}\right)_{2} \mathrm{NH}\right)_{3}$ & 5.909 & 19.762 \\
\hline$\left(\mathrm{H}_{2} \mathrm{SO}_{4}\right)_{2} \cdot\left(\left(\mathrm{CH}_{3}\right)_{2} \mathrm{NH}\right)_{3}$ & 7.030 & 24.141 \\
\hline$\left(\mathrm{H}_{2} \mathrm{SO}_{4}\right)_{3} \cdot\left(\left(\mathrm{CH}_{3}\right)_{2} \mathrm{NH}\right)_{3}$ & 6.338 & 28.669 \\
\hline$\left(\mathrm{H}_{2} \mathrm{SO}_{4}\right)_{4} \cdot\left(\left(\mathrm{CH}_{3}\right)_{2} \mathrm{NH}\right)_{3}$ & 8.378 & 33.305 \\
\hline$\left(\left(\mathrm{CH}_{3}\right)_{2} \mathrm{NH}\right)_{4}$ & 0.005 & 20.512 \\
\hline $\mathrm{H}_{2} \mathrm{SO}_{4} \cdot\left(\left(\mathrm{CH}_{3}\right)_{2} \mathrm{NH}\right)_{4}$ & 1.415 & 25.246 \\
\hline$\left(\mathrm{H}_{2} \mathrm{SO}_{4}\right)_{2} \cdot\left(\left(\mathrm{CH}_{3}\right)_{2} \mathrm{NH}\right)_{4}$ & 9.154 & 29.324 \\
\hline$\left(\mathrm{H}_{2} \mathrm{SO}_{4}\right)_{3} \cdot\left(\left(\mathrm{CH}_{3}\right)_{2} \mathrm{NH}\right)_{4}$ & 5.046 & 33.774 \\
\hline$\left(\mathrm{H}_{2} \mathrm{SO}_{4}\right)_{4} \cdot\left(\left(\mathrm{CH}_{3}\right)_{2} \mathrm{NH}\right)_{4}$ & 4.802 & 38.239 \\
\hline
\end{tabular}

a: from Sedo et al. (2008)

b: from Nadykto and $\mathrm{Yu}(2003)$

c: from CRC Handbook of Chemistry and Physics (2010)

Table S-1. Dipole moments and polarizabilities of all studied clusters at 298 K. For molecules we used experimental values, except for the sulfuric acid polarizability, for which only a theoretical value is available, and for clusters we 
used values calculated for the minimum-free energy clusters (at the corresponding temperature) with the Gaussian09 program (Frisch et al. 2009) using the B3LYP hybrid functional (Becke et al. 1993) and a CBSB7 basis set (Montgomery et al. 1999).

xyz of the most stable clusters.

Negatively charged pure sulfuric acid clusters

$\mathrm{H}_{2} \mathrm{SO}_{4} \cdot \mathrm{HSO}_{4}{ }^{-}$

13

$\begin{array}{lccc}\mathrm{S} & 1.885090 & 0.081878 & 0.000971 \\ \mathrm{O} & 1.376614 & -0.738685 & -1.240795 \\ \mathrm{O} & 1.367591 & -0.739236 & 1.236822 \\ \mathrm{O} & 3.313294 & 0.025501 & 0.004899 \\ \mathrm{O} & 1.226273 & 1.378451 & -0.001615 \\ \mathrm{H} & 0.354647 & -0.696966 & -1.276651 \\ \mathrm{H} & 0.343513 & -0.690978 & 1.273184 \\ \mathrm{~S} & -1.874131 & -0.097435 & -0.000002 \\ \mathrm{O} & -1.193334 & -0.604079 & -1.216305 \\ \mathrm{O} & -1.194390 & -0.591910 & 1.222778 \\ \mathrm{O} & -3.303785 & -0.239057 & 0.000822 \\ \mathrm{O} & -1.620295 & 1.509093 & -0.007310 \\ \mathrm{H} & -0.649250 & 1.636231 & -0.006401\end{array}$

$\left(\mathrm{H}_{2} \mathrm{SO}_{4}\right)_{2} \cdot \mathrm{HSO}_{4}{ }^{-}$

20

$\begin{array}{lccc}\mathrm{S} & -3.402718 & -0.665977 & 0.086856 \\ \mathrm{O} & -4.624573 & -1.397614 & 0.092963 \\ \mathrm{O} & -2.988707 & 0.066657 & 1.270349 \\ \mathrm{O} & -3.456725 & 0.339533 & -1.128678 \\ \mathrm{O} & -2.249106 & -1.670606 & -0.305472 \\ \mathrm{H} & -2.632864 & 0.914614 & -1.117660 \\ \mathrm{H} & -1.363831 & -1.202866 & -0.248619 \\ \mathrm{O} & -0.603486 & 1.515140 & 1.418243 \\ \mathrm{H} & -1.464583 & 1.058408 & 1.544134 \\ \mathrm{~S} & -0.134979 & 1.224480 & -0.086018 \\ \mathrm{O} & 0.006473 & -0.260567 & -0.184989 \\ \mathrm{O} & -1.202932 & 1.706251 & -0.967354 \\ \mathrm{O} & 1.127465 & 1.924658 & -0.209801 \\ \mathrm{~S} & 3.589058 & -0.609430 & 0.091543 \\ \mathrm{O} & 2.421418 & -1.372519 & -0.673792 \\ \mathrm{O} & 3.565649 & 0.833031 & -0.579684 \\ \mathrm{H} & 1.539203 & -0.997576 & -0.414300 \\ \mathrm{H} & 2.707291 & 1.283850 & -0.370979 \\ \mathrm{O} & 4.811005 & -1.222723 & -0.321655 \\ \mathrm{O} & 3.242645 & -0.491440 & 1.481036\end{array}$

$\left(\mathrm{H}_{2} \mathrm{SO}_{4}\right)_{3} \cdot \mathrm{HSO}_{4}{ }^{-}$

27

$\begin{array}{llll}\mathrm{S} & -4.582865 & -0.289105 & -0.316335 \\ \mathrm{O} & -5.969527 & -0.167189 & -0.607113 \\ \mathrm{O} & -3.749086 & -1.195850 & -1.084679\end{array}$




$\begin{array}{lccc}\mathrm{O} & -4.450593 & -0.666233 & 1.212967 \\ \mathrm{O} & -3.950038 & 1.158097 & -0.402773 \\ \mathrm{H} & -3.489368 & -0.825418 & 1.428803 \\ \mathrm{H} & -2.959424 & 1.096306 & -0.312238 \\ \mathrm{~S} & -0.903022 & -0.530220 & 0.498288 \\ \mathrm{O} & -1.030533 & -1.571147 & -0.704069 \\ \mathrm{O} & -1.811365 & -0.956269 & 1.558279 \\ \mathrm{O} & 0.518094 & -0.587895 & 0.839132 \\ \mathrm{O} & -1.295787 & 0.797087 & -0.054376 \\ \mathrm{H} & -1.975930 & -1.587092 & -0.978495 \\ \mathrm{~S} & 3.744335 & -1.650886 & -0.079470 \\ \mathrm{O} & 3.799361 & -0.741894 & -1.382224 \\ \mathrm{O} & 4.737322 & -2.658963 & -0.256511 \\ \mathrm{H} & 3.280562 & 0.085034 & -1.236922 \\ \mathrm{O} & 3.716406 & -0.796272 & 1.078477 \\ \mathrm{O} & 2.335835 & -2.380476 & -0.200774 \\ \mathrm{H} & 1.633307 & -1.801634 & 0.178442 \\ \mathrm{~S} & 1.885307 & 2.464999 & -0.089611 \\ \mathrm{O} & 2.626689 & 3.675780 & 0.003791 \\ \mathrm{O} & 2.227943 & 1.461370 & -1.077931 \\ \mathrm{O} & 1.899185 & 1.788112 & 1.342094 \\ \mathrm{O} & 0.374959 & 2.866592 & -0.299455 \\ \mathrm{H} & 1.595242 & 0.853229 & 1.274036 \\ \mathrm{H} & -0.215397 & 2.064150 & -0.278237\end{array}$

$\left(\mathrm{H}_{2} \mathrm{SO}_{4}\right)_{4} \cdot \mathrm{HSO}_{4}{ }^{-}$

34

$\begin{array}{lccc}\mathrm{S} & 3.088858 & -3.088795 & -0.257419 \\ \mathrm{~S} & -5.022801 & -0.887229 & 0.084916 \\ \mathrm{~S} & -1.247783 & -0.820358 & 0.182592 \\ \mathrm{~S} & 0.086804 & 3.614295 & -0.381960 \\ \mathrm{O} & -1.836774 & -1.784508 & -0.733723 \\ \mathrm{O} & 0.218749 & -0.794573 & 0.162562 \\ \mathrm{O} & -1.840020 & 0.536830 & 0.008899 \\ \mathrm{O} & -6.443335 & -0.887168 & 0.043808 \\ \mathrm{O} & -4.323401 & -1.186704 & 1.321350 \\ \mathrm{O} & 2.741622 & -2.788769 & 1.104960 \\ \mathrm{O} & 3.395051 & -4.409323 & -0.699055 \\ \mathrm{O} & 1.359803 & 4.104771 & 0.117541 \\ \mathrm{O} & -0.923826 & 4.549454 & -0.745200 \\ \mathrm{O} & -1.589887 & -1.263994 & 1.673141 \\ \mathrm{O} & -4.531543 & 0.526155 & -0.431270 \\ \mathrm{O} & -4.518481 & -1.889935 & -1.029171 \\ \mathrm{O} & 4.340190 & -2.198450 & -0.684177 \\ \mathrm{O} & 1.931428 & -2.533636 & -1.202954 \\ \mathrm{O} & -0.400534 & 2.605840 & 0.721048 \\ \mathrm{O} & 0.331914 & 2.692721 & -1.642744 \\ \mathrm{H} & -3.552685 & 0.616213 & -0.286714 \\ \mathrm{H} & -3.527402 & -1.954928 & -0.984791 \\ \mathrm{H} & -2.572366 & -1.300260 & 1.744363 \\ \mathrm{H} & 1.300464 & -1.979768 & -0.690383 \\ \mathrm{H} & 4.322032 & -1.333588 & -0.218468 \\ \mathrm{H} & 1.097808 & 2.088608 & -1.462554 \\ \mathrm{H} & -1.015493 & 1.889981 & 0.380694 \\ \mathrm{~S} & 3.070234 & 1.112923 & 0.390987 \\ \mathrm{O} & 3.388850 & 2.551740 & 0.909798 \\ \mathrm{O} & 2.037923 & 0.617778 & 1.459943 \\ \mathrm{O} & 4.290298 & 0.360702 & 0.486105\end{array}$




$\begin{array}{llll}\mathrm{O} & 2.421424 & 1.172478 & -0.905540 \\ \mathrm{H} & 2.628925 & 3.164450 & 0.690063 \\ \mathrm{H} & 1.318141 & 0.064655 & 1.039388\end{array}$

Negatively charged ammonia-sulfuric acid clusters

$\mathrm{HSO}_{4}^{-} \cdot \mathrm{NH}_{3}$

\begin{tabular}{|c|c|c|c|}
\hline \multicolumn{2}{|c|}{10} & & \\
\hline S & 0.609055 & -0.107742 & 0.000002 \\
\hline $\mathrm{O}$ & -0.023887 & 1.435959 & 0.000052 \\
\hline $\mathrm{O}$ & 0.074161 & -0.724944 & 1.223621 \\
\hline $\mathrm{O}$ & 0.073177 & -0.725259 & -1.223090 \\
\hline $\mathrm{O}$ & 2.040100 & 0.135704 & -0.000608 \\
\hline $\mathrm{H}$ & -0.992113 & 1.307460 & 0.000214 \\
\hline $\mathrm{N}$ & -2.596280 & 0.057730 & 0.000008 \\
\hline $\mathrm{H}$ & -2.150145 & -0.393704 & -0.798525 \\
\hline $\mathrm{H}$ & -3.584509 & -0.172057 & -0.001427 \\
\hline $\mathrm{H}$ & -2.152552 & -0.393627 & 0.799862 \\
\hline
\end{tabular}

17

$\begin{array}{lccc}\mathrm{S} & -2.104870 & -0.234201 & -0.078803 \\ \mathrm{O} & -3.404161 & -0.854328 & -0.089359 \\ \mathrm{O} & -1.167455 & -0.657974 & -1.134823 \\ \mathrm{~S} & 2.074151 & -0.238942 & 0.038329 \\ \mathrm{O} & 2.100363 & 0.879453 & -0.942730 \\ \mathrm{O} & 3.372956 & -0.728493 & 0.423075 \\ \mathrm{O} & 1.174472 & 0.125680 & 1.170172 \\ \mathrm{~N} & 0.162154 & 2.421402 & -0.006605 \\ \mathrm{H} & 0.886325 & 1.884725 & -0.576835 \\ \mathrm{O} & -1.431331 & -0.702040 & 1.317508 \\ \mathrm{H} & -0.467850 & -0.475363 & 1.298201 \\ \mathrm{H} & 0.468806 & 2.259298 & 0.953280 \\ \mathrm{H} & 0.146557 & 3.410622 & -0.231564 \\ \mathrm{O} & 1.360092 & -1.470948 & -0.700496 \\ \mathrm{H} & 0.422325 & -1.213450 & -0.908185 \\ \mathrm{O} & -2.164833 & 1.257688 & -0.011374 \\ \mathrm{H} & -0.820565 & 1.962339 & -0.096860\end{array}$

$\left(\mathrm{H}_{2} \mathrm{SO}_{4}\right)_{2} \cdot \mathrm{HSO}_{4}{ }^{-} \cdot \mathrm{NH}_{3}$

24

$\begin{array}{lccc}\mathrm{S} & 0.000023 & 1.513211 & 0.154400 \\ \mathrm{O} & 0.000007 & 0.094345 & -0.476417 \\ \mathrm{O} & 1.230499 & 2.192324 & -0.300333 \\ \mathrm{O} & -1.230490 & 2.192319 & -0.300248 \\ \mathrm{O} & 0.000098 & 1.327896 & 1.630784 \\ \mathrm{~N} & -0.000194 & -1.249107 & 2.000910 \\ \mathrm{H} & 0.000152 & -1.631407 & 2.941174 \\ \mathrm{H} & -0.860003 & -1.513810 & 1.495009 \\ \mathrm{H} & 0.859276 & -1.513823 & 1.494484 \\ \mathrm{H} & -0.000151 & -0.171144 & 1.999482 \\ \mathrm{~S} & 3.236392 & -0.672189 & -0.240863\end{array}$




$\begin{array}{lccc}\mathrm{S} & -3.236380 & -0.672171 & -0.240947 \\ \mathrm{O} & 2.610220 & -1.279322 & 0.926807 \\ \mathrm{O} & -4.485804 & -1.207253 & -0.681714 \\ \mathrm{O} & -2.610260 & -1.279974 & 0.926412 \\ \mathrm{O} & 4.485780 & -1.207546 & -0.681395 \\ \mathrm{O} & 3.434079 & 0.863768 & 0.017348 \\ \mathrm{O} & -3.433954 & 0.863677 & 0.018097 \\ \mathrm{O} & -2.219790 & -0.725995 & -1.435728 \\ \mathrm{H} & 2.563421 & 1.394848 & -0.065286 \\ \mathrm{H} & -2.563384 & 1.394811 & -0.065016 \\ \mathrm{H} & -1.305322 & -0.389230 & -1.115598 \\ \mathrm{O} & 2.219789 & -0.726573 & -1.435650 \\ \mathrm{H} & 1.305433 & -0.389449 & -1.115753\end{array}$

$\left(\mathrm{H}_{2} \mathrm{SO}_{4}\right)_{3} \cdot \mathrm{HSO}_{4} \cdot \mathrm{NH}_{3}$

31

$\begin{array}{lccc}\mathrm{N} & -0.138314 & -1.211746 & -0.331102 \\ \mathrm{H} & -0.987024 & -1.756403 & -0.133500 \\ \mathrm{H} & 0.673739 & -1.768688 & -0.622593 \\ \mathrm{H} & -0.399976 & -0.506549 & -1.058572 \\ \mathrm{~S} & -1.966329 & 1.530177 & -0.714776 \\ \mathrm{O} & -1.777175 & 0.885015 & 0.609943 \\ \mathrm{O} & -1.115270 & 0.907403 & -1.734211 \\ \mathrm{H} & 3.318653 & 3.305903 & 1.514824 \\ \mathrm{~S} & 2.226845 & 1.781744 & 0.510444 \\ \mathrm{O} & 3.076643 & 2.403100 & 1.762493 \\ \mathrm{O} & 3.189918 & 1.585973 & -0.579022 \\ \mathrm{O} & 1.759942 & 0.508982 & 1.087575 \\ \mathrm{O} & 1.192544 & 2.755275 & 0.215381 \\ \mathrm{H} & 0.124365 & -0.648596 & 0.484653 \\ \mathrm{O} & -3.376593 & 1.644355 & -1.081814 \\ \mathrm{H} & -4.295213 & 0.260941 & -0.845097 \\ \mathrm{~S} & 3.770012 & -1.910173 & -0.320729 \\ \mathrm{O} & 4.533825 & -0.661925 & -0.901553 \\ \mathrm{O} & 3.480734 & -1.509505 & 1.184251 \\ \mathrm{O} & 4.688909 & -2.998836 & -0.301066 \\ \mathrm{O} & 2.502044 & -2.051300 & -1.009511 \\ \mathrm{H} & 3.985884 & 0.177428 & -0.832165 \\ \mathrm{H} & 2.831064 & -0.748444 & 1.218283 \\ \mathrm{~S} & -4.125155 & -1.484831 & 0.419536 \\ \mathrm{O} & -3.719985 & -0.514861 & 1.592872 \\ \mathrm{O} & -5.105193 & -2.394750 & 0.914552 \\ \mathrm{O} & -4.854476 & -0.538711 & -0.614509 \\ \mathrm{O} & -2.913993 & -2.005913 & -0.188270 \\ \mathrm{H} & -2.962190 & 0.082614 & 1.284936 \\ \mathrm{O} & -1.472282 & 3.037617 & -0.546986 \\ \mathrm{H} & -0.523794 & 2.997988 & -0.285657\end{array}$

$\left(\mathrm{H}_{2} \mathrm{SO}_{4}\right)_{4} \cdot \mathrm{HSO}_{4} \cdot \mathrm{NH}_{3}$

38

$\begin{array}{lccc}\mathrm{S} & -0.398361 & -0.279180 & -0.992393 \\ \mathrm{O} & -1.882325 & -0.085948 & -1.094249 \\ \mathrm{O} & -0.083074 & -1.715667 & -1.235082 \\ \mathrm{O} & 0.289966 & 0.603805 & -1.975354 \\ \mathrm{O} & 0.045361 & 0.099631 & 0.382282 \\ \mathrm{~N} & -1.586634 & 0.724761 & 2.362541\end{array}$




$\begin{array}{lrrr}\mathrm{H} & -0.893755 & 0.839446 & 3.112831 \\ \mathrm{H} & -2.007597 & 1.615704 & 2.089316 \\ \mathrm{H} & -2.277008 & -0.000157 & 2.565865 \\ \mathrm{H} & -1.036766 & 0.393844 & 1.525653 \\ \mathrm{~S} & -2.939299 & -2.833434 & 0.687852 \\ \mathrm{~S} & 2.221409 & 0.913493 & 2.757383 \\ \mathrm{~S} & 3.381065 & -1.164331 & -1.668340 \\ \mathrm{~S} & -1.899327 & 3.331183 & -0.924860 \\ \mathrm{O} & 1.018936 & 0.781329 & 3.552485 \\ \mathrm{O} & 3.353459 & 1.570712 & 3.326369 \\ \mathrm{O} & -2.595525 & -1.828828 & 1.672469 \\ \mathrm{O} & 3.240374 & -0.571738 & -0.353597 \\ \mathrm{O} & -2.403523 & 4.641656 & -1.162052 \\ \mathrm{O} & -1.747281 & 2.848470 & 0.431518 \\ \mathrm{O} & 4.663561 & -1.558021 & -2.142623 \\ \mathrm{O} & 1.904293 & 1.641413 & 1.395450 \\ \mathrm{O} & 2.577648 & -0.562764 & 2.295053 \\ \mathrm{O} & -3.834929 & -3.886519 & 1.029955 \\ \mathrm{O} & -1.611825 & -3.512427 & 0.155125 \\ \mathrm{O} & 2.772473 & -0.160452 & -2.725423 \\ \mathrm{O} & 2.438979 & -2.427599 & -1.762370 \\ \mathrm{O} & -0.504366 & 3.235845 & -1.672062 \\ \mathrm{O} & -2.807795 & 2.310689 & -1.720039 \\ \mathrm{H} & 1.223308 & 1.119329 & 0.881304 \\ \mathrm{H} & 2.962445 & -0.575116 & 1.386854 \\ \mathrm{H} & -1.016194 & -2.855315 & -0.295596 \\ \mathrm{H} & 1.883444 & 0.181629 & -2.430325 \\ \mathrm{H} & 1.500032 & -2.189479 & -1.516761 \\ \mathrm{H} & -0.180977 & 2.299425 & -1.747563 \\ \mathrm{H} & -2.515211 & 1.376740 & -1.509386 \\ \mathrm{O} & -3.515071 & -2.104282 & -0.590347 \\ \mathrm{H} & -2.937755 & -1.317491 & -0.814328 \\ & & & \end{array}$

$\left(\mathrm{H}_{2} \mathrm{SO}_{4}\right)_{2} \cdot \mathrm{HSO}_{4} \cdot \cdot\left(\mathrm{NH}_{3}\right)_{2}$

28

$\begin{array}{lrrr}\mathrm{S} & 3.127914 & -1.027915 & 0.067332 \\ \mathrm{O} & 2.787773 & -0.606030 & 1.443234 \\ \mathrm{O} & 3.087422 & 0.334199 & -0.825501 \\ \mathrm{O} & 4.462341 & -1.535899 & -0.111367 \\ \mathrm{H} & 2.295796 & 0.862091 & -0.578605 \\ \mathrm{~S} & -2.711515 & -1.269772 & 0.009879 \\ \mathrm{O} & -3.055582 & 0.127075 & 0.223859 \\ \mathrm{O} & -4.130351 & -2.033761 & 0.319086 \\ \mathrm{O} & -2.378049 & -1.623274 & -1.377431 \\ \mathrm{H} & -3.953825 & -2.978363 & 0.216328 \\ \mathrm{~S} & -0.273991 & 2.545442 & -0.030039 \\ \mathrm{O} & 0.564672 & 1.422081 & -0.560176 \\ \mathrm{O} & -0.494004 & 2.347660 & 1.413277 \\ \mathrm{O} & -1.708768 & 2.346646 & -0.741687 \\ \mathrm{O} & 0.166167 & 3.850516 & -0.440443 \\ \mathrm{O} & 2.058403 & -1.891898 & -0.492396 \\ \mathrm{~N} & 0.222846 & -0.191407 & 2.162152 \\ \mathrm{~N} & 0.202111 & -0.793559 & -2.048821 \\ \mathrm{H} & -2.179934 & 1.581123 & -0.339749 \\ \mathrm{H} & 0.950995 & -1.314920 & -1.503071 \\ \mathrm{H} & 0.420891 & -0.783559 & -3.040016 \\ \mathrm{H} & -0.736849 & -1.206365 & -1.877601 \\ \mathrm{H} & 0.208247 & 0.178904 & -1.663852\end{array}$




$\begin{array}{lrrr}\mathrm{H} & 1.211277 & -0.400995 & 1.845820 \\ \mathrm{H} & -0.455844 & -0.834092 & 1.711707 \\ \mathrm{H} & -0.028277 & 0.796067 & 1.902643 \\ \mathrm{H} & 0.160612 & -0.300663 & 3.170305 \\ \mathrm{O} & -1.753313 & -1.820882 & 0.975550\end{array}$

$\left(\mathrm{H}_{2} \mathrm{SO}_{4}\right)_{3} \cdot \mathrm{HSO}_{4}{ }^{-} \cdot\left(\mathrm{NH}_{3}\right)_{2}$

35

$\begin{array}{lccc}\mathrm{N} & -1.735395 & 2.146441 & 0.405698 \\ \mathrm{H} & -0.909969 & 2.472780 & -0.159418 \\ \mathrm{H} & -2.625573 & 2.132945 & -0.110115 \\ \mathrm{H} & -1.814970 & 2.720009 & 1.241075 \\ \mathrm{~N} & 1.768085 & -1.297360 & 1.675908 \\ \mathrm{H} & 2.727479 & -1.500277 & 1.244280 \\ \mathrm{H} & 1.752410 & -1.599542 & 2.645613 \\ \mathrm{H} & 1.576918 & -0.275062 & 1.621808 \\ \mathrm{~S} & 1.533841 & 1.733421 & -0.132778 \\ \mathrm{O} & 0.652157 & 2.724460 & -0.755459 \\ \mathrm{O} & 1.635771 & 0.469284 & -0.926212 \\ \mathrm{O} & 1.128409 & 1.418629 & 1.251635 \\ \mathrm{H} & 0.140709 & -0.206706 & -1.251569 \\ \mathrm{~S} & -1.379821 & -1.455650 & -0.310346 \\ \mathrm{O} & -0.812928 & -0.465624 & -1.419229 \\ \mathrm{O} & -2.554074 & -2.058049 & -0.933680 \\ \mathrm{O} & -0.322420 & -2.379538 & 0.067754 \\ \mathrm{O} & -1.778959 & -0.578586 & 0.843882 \\ \mathrm{H} & -1.558398 & 1.157417 & 0.683744 \\ \mathrm{H} & 1.030135 & -1.788347 & 1.141169 \\ \mathrm{O} & 2.956137 & 2.380598 & -0.126172 \\ \mathrm{H} & 3.638832 & 1.697309 & 0.226038 \\ \mathrm{~S} & -5.048564 & 0.178716 & 0.072126 \\ \mathrm{O} & -4.995201 & -1.077922 & -0.870422 \\ \mathrm{O} & -4.331231 & -0.311089 & 1.395884 \\ \mathrm{O} & -6.416129 & 0.423053 & 0.389296 \\ \mathrm{O} & -4.252957 & 1.244421 & -0.510944 \\ \mathrm{H} & -4.054744 & -1.420910 & -0.968438 \\ \mathrm{H} & -3.353482 & -0.477363 & 1.222483 \\ \mathrm{~S} & 4.858812 & -0.646932 & 0.019295 \\ \mathrm{O} & 4.270633 & -0.403051 & -1.470719 \\ \mathrm{O} & 6.266144 & -0.857387 & -0.171452 \\ \mathrm{O} & 4.493231 & 0.576109 & 0.785498 \\ \mathrm{O} & 4.120500 & -1.810205 & 0.564530 \\ \mathrm{H} & 3.320885 & -0.169539 & -1.394181\end{array}$

$\left(\mathrm{H}_{2} \mathrm{SO}_{4}\right)_{4} \cdot \mathrm{HSO}_{4} \cdot\left(\mathrm{NH}_{3}\right)_{2}$

42

$\begin{array}{lrrr}\mathrm{S} & 2.688606 & 0.187607 & 1.430394 \\ \mathrm{O} & 3.140529 & 0.298179 & -0.031330 \\ \mathrm{O} & 3.314099 & -1.022178 & 2.016408 \\ \mathrm{O} & 3.043640 & 1.423468 & 2.129571 \\ \mathrm{O} & 1.191171 & -0.003882 & 1.402338 \\ \mathrm{~N} & 0.289159 & 0.352874 & -1.147663 \\ \mathrm{H} & -0.738391 & 0.302892 & -1.229478 \\ \mathrm{H} & 0.668697 & 1.249132 & -1.478628 \\ \mathrm{H} & 0.730666 & -0.449212 & -1.605828 \\ \mathrm{H} & 0.559740 & 0.291748 & -0.136192\end{array}$




$\begin{array}{lccc}\mathrm{S} & 2.936508 & -2.920665 & -0.869461 \\ \mathrm{~S} & -2.807238 & -1.374613 & -0.328614 \\ \mathrm{~S} & -5.706033 & 0.985338 & 0.190763 \\ \mathrm{~S} & 2.660667 & 3.611922 & -0.623851 \\ \mathrm{O} & -2.565757 & -0.011565 & -0.872629 \\ \mathrm{O} & -1.680604 & -2.269184 & -0.547005 \\ \mathrm{O} & 1.646278 & -2.332180 & -1.181573 \\ \mathrm{O} & -4.970816 & 0.512780 & 1.350764 \\ \mathrm{O} & 2.967216 & 4.883738 & -1.189381 \\ \mathrm{O} & 1.436676 & 2.937947 & -1.017804 \\ \mathrm{O} & -6.797488 & 1.882384 & 0.350442 \\ \mathrm{O} & -4.114053 & -1.895863 & -0.720730 \\ \mathrm{O} & -2.819271 & -1.208132 & 1.279687 \\ \mathrm{O} & 3.333710 & -4.124433 & -1.521844 \\ \mathrm{O} & 2.949255 & -3.199891 & 0.708041 \\ \mathrm{O} & -6.240555 & -0.273116 & -0.604013 \\ \mathrm{O} & -4.679588 & 1.653268 & -0.807989 \\ \mathrm{O} & 2.675472 & 3.779508 & 0.944599 \\ \mathrm{O} & 3.874362 & 2.644589 & -0.904882 \\ \mathrm{H} & -3.550470 & -0.587427 & 1.502700 \\ \mathrm{H} & 3.170172 & -2.342754 & 1.229164 \\ \mathrm{H} & -5.512340 & -0.952269 & -0.670596 \\ \mathrm{H} & -3.849619 & 1.100564 & -0.856494 \\ \mathrm{H} & 2.749211 & 2.894570 & 1.412606 \\ \mathrm{H} & 3.644577 & 1.717702 & -0.579966 \\ \mathrm{O} & 4.054516 & -1.846129 & -1.048875 \\ \mathrm{H} & 3.735201 & -0.947964 & -0.659244 \\ \mathrm{~N} & 0.173153 & -2.481229 & 1.500145 \\ \mathrm{H} & -0.282759 & -2.765073 & 2.363112 \\ \mathrm{H} & 0.966181 & -3.092569 & 1.277250 \\ \mathrm{H} & 0.559023 & -1.495644 & 1.588938 \\ \mathrm{H} & -0.516569 & -2.493095 & 0.717240\end{array}$

$\left(\mathrm{H}_{2} \mathrm{SO}_{4}\right)_{3} \cdot \mathrm{HSO}_{4}{ }^{-} \cdot\left(\mathrm{NH}_{3}\right)_{3}$

39

$\begin{array}{lccc}\mathrm{S} & 1.922290 & 2.518457 & -0.167115 \\ \mathrm{O} & 2.268476 & 2.360138 & 1.248310 \\ \mathrm{O} & 3.277413 & 3.113572 & -0.866386 \\ \mathrm{O} & 1.727308 & 1.258309 & -0.892224 \\ \mathrm{H} & 3.685512 & 3.701832 & -0.217607 \\ \mathrm{~S} & -3.792065 & -0.186795 & -1.256514 \\ \mathrm{O} & -2.523239 & 0.218022 & -1.936585 \\ \mathrm{O} & -3.593979 & -1.732983 & -0.853922 \\ \mathrm{O} & -3.913664 & 0.557778 & 0.022576 \\ \mathrm{H} & -2.935713 & -1.791154 & -0.120435 \\ \mathrm{~S} & -1.425009 & -0.616543 & 1.994557 \\ \mathrm{O} & -0.760218 & -0.980550 & 3.235221 \\ \mathrm{O} & -1.631704 & -1.739633 & 1.052770 \\ \mathrm{O} & -0.740437 & 0.512064 & 1.320956 \\ \mathrm{O} & -2.877600 & -0.103839 & 2.406723 \\ \mathrm{H} & -3.370244 & 0.107351 & 1.564576 \\ \mathrm{O} & 0.869313 & 3.501779 & -0.418149 \\ \mathrm{~N} & 1.962830 & -0.230198 & 2.395817 \\ \mathrm{~N} & -1.672350 & 2.347528 & -0.429710 \\ \mathrm{~N} & -0.034016 & -1.050659 & -1.256218 \\ \mathrm{H} & -1.455511 & 1.721274 & 0.379978 \\ \mathrm{H} & -1.961887 & 1.694904 & -1.194756 \\ \mathrm{H} & -2.457661 & 2.948086 & -0.197081\end{array}$




$\begin{array}{llll}\mathrm{H} & -0.811671 & 2.885783 & -0.634580 \\ \mathrm{H} & -0.840116 & -0.826959 & -1.854046 \\ \mathrm{H} & -0.387619 & -1.525124 & -0.413083 \\ \mathrm{H} & 0.728006 & -1.612705 & -1.698859 \\ \mathrm{H} & 0.399784 & -0.169842 & -0.951336 \\ \mathrm{H} & 1.038550 & -0.437729 & 2.811180 \\ \mathrm{H} & 1.971350 & 0.734279 & 2.013570 \\ \mathrm{H} & 2.145545 & -0.919936 & 1.600190 \\ \mathrm{H} & 2.692300 & -0.326160 & 3.096804 \\ \mathrm{O} & -4.952795 & -0.159656 & -2.102131 \\ \mathrm{~S} & 3.150272 & -2.075722 & -0.734465 \\ \mathrm{O} & 3.881067 & -0.618395 & -0.796329 \\ \mathrm{O} & 4.236285 & -3.005701 & -0.581348 \\ \mathrm{O} & 2.234202 & -1.986488 & 0.438322 \\ \mathrm{O} & 2.359438 & -2.186657 & -1.972203 \\ \mathrm{H} & 3.197376 & 0.056977 & -0.981957\end{array}$

$\left(\mathrm{H}_{2} \mathrm{SO}_{4}\right)_{4} \cdot \mathrm{HSO}_{4}{ }^{-} \cdot\left(\mathrm{NH}_{3}\right)_{3}$

46

$\begin{array}{lccc}\mathrm{S} & 1.425019 & 0.237449 & 0.343385 \\ \mathrm{O} & 2.738616 & 0.872906 & -0.008580 \\ \mathrm{O} & 1.649751 & -0.958267 & 1.195797 \\ \mathrm{O} & 0.578604 & 1.230460 & 1.090070 \\ \mathrm{O} & 0.700476 & -0.163004 & -0.898662 \\ \mathrm{~N} & -1.466054 & 1.587543 & -1.280367 \\ \mathrm{H} & -2.204812 & 1.570275 & -0.538088 \\ \mathrm{H} & -0.893109 & 2.444618 & -1.225134 \\ \mathrm{H} & -1.921985 & 1.518761 & -2.186891 \\ \mathrm{H} & -0.818004 & 0.782025 & -1.162891 \\ \mathrm{~S} & 4.604459 & -2.177395 & 0.091981 \\ \mathrm{~S} & -2.426363 & -2.597702 & -0.542434 \\ \mathrm{~S} & -4.661331 & 0.665472 & 0.393273 \\ \mathrm{~S} & 1.531680 & 4.180372 & -0.368881 \\ \mathrm{O} & -2.487335 & -1.298852 & -1.245742 \\ \mathrm{O} & -1.978023 & -3.703736 & -1.367635 \\ \mathrm{O} & 3.587163 & -2.786751 & -0.747147 \\ \mathrm{O} & -3.373245 & 1.373186 & 0.715877 \\ \mathrm{O} & 1.791460 & 5.580321 & -0.391080 \\ \mathrm{O} & 0.498365 & 3.633950 & -1.231322 \\ \mathrm{O} & -5.828869 & 1.482575 & 0.554938 \\ \mathrm{O} & -1.628934 & -2.479057 & 0.710240 \\ \mathrm{O} & 5.872993 & -2.812973 & 0.219186 \\ \mathrm{O} & 4.009131 & -1.976105 & 1.536503 \\ \mathrm{O} & -4.668332 & -0.643938 & 1.073652 \\ \mathrm{O} & -4.562082 & 0.357179 & -1.188536 \\ \mathrm{O} & 1.212959 & 3.765232 & 1.118545 \\ \mathrm{O} & 2.891236 & 3.444361 & -0.679164 \\ \mathrm{H} & 3.085024 & -1.571744 & 1.469903 \\ \mathrm{H} & -3.865663 & -0.339907 & -1.308876 \\ \mathrm{H} & 0.963153 & 2.789957 & 1.156375 \\ \mathrm{H} & 2.821877 & 2.462167 & -0.491622 \\ \mathrm{O} & 4.867413 & -0.704752 & -0.412794 \\ \mathrm{H} & 4.055168 & -0.127682 & -0.315099 \\ \mathrm{~N} & 0.755438 & -2.904988 & -0.788230 \\ \mathrm{H} & 0.333946 & -2.950932 & 0.146340 \\ \mathrm{H} & 1.748544 & -3.172664 & -0.779157 \\ \mathrm{H} & 0.705582 & -1.890609 & -1.040734 \\ \mathrm{H} & 0.154208 & -3.461391 & -1.402501 \\ & & & \\ \mathrm{H} & & \end{array}$




$\begin{array}{llll}\mathrm{N} & -1.643101 & -0.159118 & 2.157094 \\ \mathrm{H} & -1.654754 & -1.079453 & 1.648142 \\ \mathrm{H} & -0.738032 & 0.314836 & 1.980750 \\ \mathrm{H} & -1.795022 & -0.307054 & 3.150328 \\ \mathrm{H} & -2.404202 & 0.438614 & 1.748584 \\ \mathrm{O} & -3.918583 & -2.946026 & -0.107146 \\ \mathrm{H} & -4.293433 & -2.148686 & 0.357901\end{array}$

$\left(\mathrm{H}_{2} \mathrm{SO}_{4}\right)_{4} \cdot \mathrm{HSO}_{4}{ }^{-} \cdot\left(\mathrm{NH}_{3}\right)_{4}$

50

\begin{tabular}{lccc}
$\mathrm{S}$ & 1.791947 & 0.312436 & 0.657576 \\
$\mathrm{O}$ & 3.099840 & 0.937929 & 0.271608 \\
$\mathrm{O}$ & 2.021131 & -0.905236 & 1.474567 \\
$\mathrm{O}$ & 0.970364 & 1.297686 & 1.431354 \\
$\mathrm{O}$ & 1.033578 & -0.064542 & -0.577414 \\
$\mathrm{~N}$ & -1.234392 & 1.159936 & -1.530995 \\
$\mathrm{H}$ & -1.853924 & 1.598710 & -0.829858 \\
$\mathrm{H}$ & -1.020221 & 1.825173 & -2.269883 \\
$\mathrm{H}$ & -1.723107 & 0.295841 & -1.841370 \\
$\mathrm{H}$ & -0.329422 & 0.868439 & -1.098133 \\
$\mathrm{~S}$ & 4.906759 & -2.149355 & 0.162052 \\
$\mathrm{~S}$ & -2.312003 & -2.473296 & -0.910038 \\
$\mathrm{~S}$ & -4.539735 & 1.097800 & 0.615894 \\
$\mathrm{~S}$ & 1.616244 & 4.028929 & -0.650881 \\
$\mathrm{O}$ & -2.754188 & -1.123278 & -1.438286 \\
$\mathrm{O}$ & -1.423397 & -3.132478 & -1.881548 \\
$\mathrm{O}$ & 3.866225 & -2.656657 & -0.711523 \\
$\mathrm{O}$ & -3.160883 & 1.647734 & 0.509404 \\
$\mathrm{O}$ & 1.741823 & 5.423447 & -0.921296 \\
$\mathrm{O}$ & 0.694274 & 3.220922 & -1.419364 \\
$\mathrm{O}$ & -5.588693 & 2.072889 & 0.727410 \\
$\mathrm{O}$ & -1.590270 & -2.264944 & 0.388133 \\
$\mathrm{O}$ & 6.162399 & -2.822665 & 0.220651 \\
$\mathrm{O}$ & 4.336494 & -2.061165 & 1.629405 \\
$\mathrm{O}$ & -4.551409 & 0.030482 & 1.651798 \\
$\mathrm{O}$ & -4.819186 & 0.333848 & -0.780836 \\
$\mathrm{O}$ & 1.267379 & 3.872212 & 0.883497 \\
$\mathrm{O}$ & 3.065574 & 3.405870 & -0.763632 \\
$\mathrm{H}$ & 3.431884 & -1.608886 & 1.619300 \\
$\mathrm{H}$ & -4.031731 & -0.248105 & -1.017995 \\
$\mathrm{H}$ & 1.122722 & 2.905773 & 1.11683 \\
$\mathrm{H}$ & 3.074736 & 2.459572 & -0.438890 \\
$\mathrm{O}$ & 5.203619 & -0.644033 & -0.212480 \\
$\mathrm{H}$ & 4.397335 & -0.063543 & -0.083005 \\
$\mathrm{~N}$ & 1.000986 & -2.795961 & -0.683937 \\
$\mathrm{H}$ & 0.704407 & -2.990516 & 0.271164 \\
$\mathrm{H}$ & 1.939564 & -3.161591 & -0.861726 \\
$\mathrm{H}$ & 1.045320 & -1.752501 & -0.746806 \\
$\mathrm{H}$ & 0.231009 & -3.123411 & -1.308822 \\
$\mathrm{~N}$ & -1.480760 & -0.042954 & 1.936806 \\
$\mathrm{H}$ & -1.511295 & -0.908166 & 1.326739 \\
$\mathrm{H}$ & -0.537902 & 0.391628 & 1.921992 \\
$\mathrm{H}$ & -1.756758 & -0.287639 & 2.883183 \\
$\mathrm{H}$ & -2.166929 & 0.638445 & 1.548128 \\
$\mathrm{O}$ & -3.539961 & -3.302988 & -0.674028 \\
$\mathrm{~N}$ & -5.527842 & -2.211519 & 0.488939 \\
$\mathrm{H}$ & -6.119885 & -1.807844 & -0.234012 \\
$\mathrm{H}$ & -4.644371 & -2.700227 & -0.001437 \\
& & & \\
\hline
\end{tabular}




$\begin{array}{llll}\mathrm{H} & -5.190117 & -1.405282 & 1.076051 \\ \mathrm{H} & -6.056345 & -2.876864 & 1.045009\end{array}$

\section{Negatively charged DMA-sulfuric acid clusters}

$\mathrm{HSO}_{4}^{-} \cdot\left(\mathrm{CH}_{3}\right)_{2} \mathrm{NH}$

16

$\begin{array}{lccc}\mathrm{S} & 1.395645 & -0.001320 & -0.092674 \\ \mathrm{~N} & -1.955967 & -0.125586 & 0.081676 \\ \mathrm{C} & -3.147999 & -0.938360 & -0.063589 \\ \mathrm{C} & -2.204891 & 1.309537 & 0.036356 \\ \mathrm{H} & -1.254217 & -0.366278 & -0.625464 \\ \mathrm{O} & 0.615150 & -0.565433 & -1.214303 \\ \mathrm{O} & 1.215531 & 1.446875 & 0.044165 \\ \mathrm{O} & 2.763919 & -0.475243 & -0.000335 \\ \mathrm{O} & 0.683504 & -0.658752 & 1.261596 \\ \mathrm{H} & -2.869757 & -1.995036 & -0.098139 \\ \mathrm{H} & -3.813699 & -0.795276 & 0.797056 \\ \mathrm{H} & -3.737273 & -0.709721 & -0.973029 \\ \mathrm{H} & -2.774977 & 1.625491 & -0.857819 \\ \mathrm{H} & -2.777465 & 1.620887 & 0.919370 \\ \mathrm{H} & -1.242062 & 1.823264 & 0.040869 \\ \mathrm{H} & -0.276595 & -0.509749 & 1.142617\end{array}$

$\mathrm{H}_{2} \mathrm{SO}_{4} \cdot \mathrm{HSO}_{4}^{-} \cdot\left(\mathrm{CH}_{3}\right)_{2} \mathrm{NH}$

23

$\begin{array}{lrrr}\mathrm{S} & 2.067402 & -0.674397 & -0.004779 \\ \mathrm{O} & 3.349483 & -1.287511 & -0.242497 \\ \mathrm{O} & 1.169065 & -0.579099 & -1.181691 \\ \mathrm{O} & 1.321448 & -1.652628 & 1.035871 \\ \mathrm{O} & 2.140075 & 0.653403 & 0.667340 \\ \mathrm{H} & 0.394911 & -1.319463 & 1.160862 \\ \mathrm{~S} & -2.067389 & -0.674336 & 0.004724 \\ \mathrm{O} & -2.140195 & 0.653376 & -0.667476 \\ \mathrm{O} & -3.349385 & -1.287522 & 0.242700 \\ \mathrm{O} & -1.168801 & -0.579017 & 1.181464 \\ \mathrm{O} & -1.321596 & -1.652670 & -1.036009 \\ \mathrm{H} & -0.395123 & -1.319515 & -1.161196 \\ \mathrm{~N} & 0.000111 & 2.079293 & -0.000091 \\ \mathrm{C} & 0.467634 & 2.863600 & -1.159497 \\ \mathrm{H} & -0.350288 & 3.468308 & -1.555625 \\ \mathrm{H} & 0.808946 & 2.155885 & -1.913509 \\ \mathrm{H} & 1.298692 & 3.505136 & -0.861397 \\ \mathrm{C} & -0.467834 & 2.862376 & 1.159988 \\ \mathrm{H} & -1.299309 & 3.503640 & 0.862467 \\ \mathrm{H} & 0.349752 & 3.467245 & 1.556565 \\ \mathrm{H} & -0.808668 & 2.153800 & 1.913402 \\ \mathrm{H} & -0.804804 & 1.453535 & -0.307106 \\ \mathrm{H} & 0.805343 & 1.453595 & 0.306505\end{array}$

$\left(\mathrm{H}_{2} \mathrm{SO}_{4}\right)_{2} \cdot \mathrm{HSO}_{4}^{-} \cdot\left(\mathrm{CH}_{3}\right)_{2} \mathrm{NH}$ 


$\begin{array}{cccc}\mathrm{S} & -3.688160 & -0.064763 & 0.040689 \\ \mathrm{O} & -5.093232 & -0.259215 & 0.274189 \\ \mathrm{O} & -2.786073 & -0.483190 & 1.142546 \\ \mathrm{O} & -3.333365 & -1.046920 & -1.198112 \\ \mathrm{O} & -3.324033 & 1.303025 & -0.413863 \\ \mathrm{H} & -2.357824 & -1.034379 & -1.316973 \\ \mathrm{~S} & 0.179332 & -1.355944 & 0.003929 \\ \mathrm{O} & 0.603856 & -0.192880 & 0.854169 \\ \mathrm{O} & 1.300004 & -2.245220 & -0.274652 \\ \mathrm{O} & -0.555583 & -0.876942 & -1.170719 \\ \mathrm{O} & -0.824189 & -2.200499 & 0.883738 \\ \mathrm{H} & -1.641392 & -1.627742 & 1.037744 \\ \mathrm{~N} & -0.799253 & 2.003685 & 0.009532 \\ \mathrm{C} & -0.804591 & 3.043292 & 1.060922 \\ \mathrm{H} & 0.219973 & 3.317276 & 1.315565 \\ \mathrm{H} & -1.309036 & 2.637903 & 1.937039 \\ \mathrm{H} & -1.347050 & 3.919392 & 0.704025 \\ \mathrm{C} & -0.140686 & 2.394061 & -1.262269 \\ \mathrm{H} & 0.919475 & 2.574236 & -1.085356 \\ \mathrm{H} & -0.629020 & 3.282773 & -1.664199 \\ \mathrm{H} & -0.242852 & 1.559436 & -1.952392 \\ \mathrm{H} & -0.308182 & 1.157976 & 0.375865 \\ \mathrm{H} & -1.805173 & 1.703455 & -0.179188 \\ \mathrm{~S} & 3.841181 & 0.242563 & -0.052924 \\ \mathrm{O} & 3.141220 & 0.212095 & 1.369036 \\ \mathrm{O} & 3.797358 & -1.275659 & -0.507768 \\ \mathrm{O} & 3.035721 & 1.037302 & -0.948245 \\ \mathrm{O} & 5.216882 & 0.564156 & 0.159001 \\ \mathrm{H} & 2.158254 & 0.047872 & 1.242448 \\ \mathrm{H} & 2.863057 & -1.626210 & -0.494877\end{array}$

\section{$\left(\mathrm{H}_{2} \mathrm{SO}_{4}\right)_{3} \cdot \mathrm{HSO}_{4}^{-} \cdot\left(\mathrm{CH}_{3}\right)_{2} \mathrm{NH}$}

37

$\begin{array}{lccc}\mathrm{S} & -1.511574 & -3.269509 & -0.589475 \\ \mathrm{O} & -1.989421 & -4.500308 & -1.127431 \\ \mathrm{O} & -1.271403 & -2.267084 & -1.777663 \\ \mathrm{O} & -2.317120 & -2.593337 & 0.419318 \\ \mathrm{H} & -1.000474 & -1.359293 & -1.408074 \\ \mathrm{~S} & 0.336544 & -0.001044 & 0.455115 \\ \mathrm{O} & -0.365071 & -0.001111 & 1.733102 \\ \mathrm{O} & 1.180258 & 1.223721 & 0.272339 \\ \mathrm{O} & -0.697076 & -0.000143 & -0.682590 \\ \mathrm{O} & 1.179060 & -1.226398 & 0.271570 \\ \mathrm{~N} & -3.076644 & 0.003970 & 1.121689 \\ \mathrm{C} & -4.290463 & 0.001755 & 0.269249 \\ \mathrm{H} & -4.869255 & -0.897772 & 0.473497 \\ \mathrm{H} & -3.972843 & 0.004012 & -0.771630 \\ \mathrm{H} & -4.874361 & 0.897453 & 0.475935 \\ \mathrm{C} & -3.331834 & 0.002296 & 2.583927 \\ \mathrm{H} & -2.363658 & 0.003586 & 3.078374 \\ \mathrm{H} & -3.892735 & -0.894375 & 2.846470 \\ \mathrm{H} & -3.896095 & 0.896414 & 2.848022 \\ \mathrm{H} & -2.505819 & -0.824796 & 0.883766 \\ \mathrm{H} & -2.509355 & 0.835632 & 0.884547 \\ \mathrm{O} & -0.074062 & -3.525366 & -0.009536 \\ \mathrm{H} & 0.411950 & -2.656650 & 0.162847 \\ \mathrm{~S} & -1.505551 & 3.271753 & -0.589392 \\ \mathrm{O} & -2.311436 & 2.598866 & 0.421450\end{array}$




$\begin{array}{lccc}\mathrm{O} & -1.980976 & 4.503342 & -1.127650 \\ \mathrm{O} & -0.066450 & 3.523692 & -0.012230 \\ \mathrm{O} & -1.270785 & 2.267479 & -1.777108 \\ \mathrm{H} & 0.417035 & 2.653330 & 0.160705 \\ \mathrm{H} & -0.999928 & 1.359644 & -1.407619 \\ \mathrm{~S} & 4.527291 & -0.002283 & 0.018705 \\ \mathrm{O} & 4.184273 & -0.000730 & -1.376445 \\ \mathrm{O} & 5.889780 & -0.003405 & 0.448596 \\ \mathrm{O} & 3.832294 & -1.244471 & 0.729068 \\ \mathrm{O} & 3.833534 & 1.239215 & 0.731554 \\ \mathrm{H} & 2.868326 & 1.268137 & 0.497262 \\ \mathrm{H} & 2.866938 & -1.271779 & 0.495015\end{array}$

$\mathrm{H}_{2} \mathrm{SO}_{4} \cdot \mathrm{HSO}_{4}^{-} \cdot\left(\left(\mathrm{CH}_{3}\right)_{2} \mathrm{NH}\right)_{2}$

33

$\begin{array}{lccc}\mathrm{S} & 2.246270 & -1.118055 & -0.091347 \\ \mathrm{O} & 3.479829 & -1.637966 & -0.632822 \\ \mathrm{O} & 2.408002 & 0.070267 & 0.778271 \\ \mathrm{O} & 1.425294 & -0.597375 & -1.387735 \\ \mathrm{O} & 1.382531 & -2.136000 & 0.550034 \\ \mathrm{H} & 0.535121 & -0.222903 & -1.129665 \\ \mathrm{~S} & -2.073537 & 1.035868 & -0.179234 \\ \mathrm{O} & -2.559434 & -0.065087 & 0.743831 \\ \mathrm{O} & -3.154741 & 1.548301 & -1.006567 \\ \mathrm{O} & -0.954448 & 0.456491 & -1.014034 \\ \mathrm{O} & -1.474325 & 2.129762 & 0.683135 \\ \mathrm{~N} & -1.316693 & -2.257056 & 0.384118 \\ \mathrm{C} & -1.700735 & -3.187467 & 1.459114 \\ \mathrm{H} & -2.783965 & -3.323120 & 1.456556 \\ \mathrm{H} & -1.397121 & -2.757682 & 2.413531 \\ \mathrm{H} & -1.204751 & -4.151586 & 1.325257 \\ \mathrm{C} & -1.656316 & -2.715925 & -0.978529 \\ \mathrm{H} & -2.733070 & -2.882990 & -1.044810 \\ \mathrm{H} & -1.115465 & -3.635594 & -1.213093 \\ \mathrm{H} & -1.374184 & -1.922842 & -1.668465 \\ \mathrm{H} & -1.822106 & -1.275159 & 0.550239 \\ \mathrm{H} & -0.281642 & -2.102320 & 0.435913 \\ \mathrm{~N} & 1.039369 & 2.378187 & 0.389745 \\ \mathrm{H} & 1.508779 & 1.446672 & 0.491147 \\ \mathrm{H} & -0.059433 & 2.221422 & 0.523139 \\ \mathrm{C} & 1.263895 & 2.889488 & -0.977945 \\ \mathrm{C} & 1.530364 & 3.260314 & 1.462208 \\ \mathrm{H} & 1.006620 & 4.217184 & 1.417784 \\ \mathrm{H} & 1.325079 & 2.785105 & 2.421330 \\ \mathrm{H} & 2.606542 & 3.420320 & 1.362929 \\ \mathrm{H} & 2.333982 & 2.990373 & -1.173474 \\ \mathrm{H} & 0.767752 & 3.855940 & -1.086052 \\ \mathrm{H} & 0.820501 & 2.178651 & -1.672010\end{array}$

$\left(\mathrm{H}_{2} \mathrm{SO}_{4}\right)_{2} \cdot \mathrm{HSO}_{4} \cdot \cdot\left(\left(\mathrm{CH}_{3}\right)_{2} \mathrm{NH}\right)_{2}$

40

$\begin{array}{llll}\mathrm{S} & -3.332236 & 1.073716 & -0.076425 \\ \mathrm{O} & -4.763740 & 1.153412 & -0.224479 \\ \mathrm{O} & -2.653531 & 2.331232 & 0.293352 \\ \mathrm{O} & -3.106968 & 0.052539 & 1.163445 \\ \mathrm{O} & -2.629375 & 0.433924 & -1.225157\end{array}$




$\begin{array}{lccc}\mathrm{H} & -2.154787 & 0.074177 & 1.465602 \\ \mathrm{~S} & 0.381113 & -1.023207 & 1.269494 \\ \mathrm{O} & -0.292459 & -2.345827 & 1.184324 \\ \mathrm{O} & 1.677492 & -1.114239 & 1.973649 \\ \mathrm{O} & -0.509958 & -0.007229 & 1.886332 \\ \mathrm{O} & 0.642132 & -0.549196 & -0.176275 \\ \mathrm{~N} & -1.995228 & -2.152019 & -0.860398 \\ \mathrm{C} & -1.263981 & -2.639378 & -2.049717 \\ \mathrm{H} & -1.046546 & -3.702447 & -1.936569 \\ \mathrm{H} & -0.331100 & -2.082507 & -2.112411 \\ \mathrm{H} & -1.864491 & -2.474058 & -2.945681 \\ \mathrm{C} & -3.277361 & -2.833909 & -0.579683 \\ \mathrm{H} & -3.096562 & -3.893453 & -0.392108 \\ \mathrm{H} & -3.951312 & -2.712683 & -1.428867 \\ \mathrm{H} & -3.716186 & -2.364329 & 0.299088 \\ \mathrm{H} & -1.351825 & -2.243372 & -0.016673 \\ \mathrm{H} & -2.193092 & -1.120680 & -0.981549 \\ \mathrm{~N} & 0.025305 & 2.141933 & -0.207528 \\ \mathrm{H} & -1.009554 & 2.220230 & -0.052054 \\ \mathrm{H} & 0.247761 & 1.117604 & -0.174787 \\ \mathrm{C} & 0.728032 & 2.823681 & 0.910304 \\ \mathrm{C} & 0.371071 & 2.645237 & -1.557101 \\ \mathrm{H} & 1.431468 & 2.469514 & -1.734439 \\ \mathrm{H} & -0.235427 & 2.103000 & -2.281523 \\ \mathrm{H} & 0.143237 & 3.710500 & -1.617073 \\ \mathrm{H} & 0.453034 & 3.879731 & 0.911235 \\ \mathrm{H} & 1.801142 & 2.699177 & 0.778163 \\ \mathrm{H} & 0.405595 & 2.345975 & 1.833168 \\ \mathrm{~S} & 3.914454 & 0.087782 & -0.469953 \\ \mathrm{O} & 5.213348 & -0.068864 & -1.050909 \\ \mathrm{O} & 3.315513 & 1.409148 & -0.444406 \\ \mathrm{O} & 3.983783 & -0.481650 & 0.995882 \\ \mathrm{O} & 2.930373 & -0.896247 & -1.203586 \\ \mathrm{H} & 1.985660 & -0.793179 & -0.800944 \\ \mathrm{H} & 3.059683 & -0.685060 & 1.372858\end{array}$

\section{$\left(\mathrm{H}_{2} \mathrm{SO}_{4}\right)_{3} \cdot \mathrm{HSO}_{4} \cdot\left(\left(\mathrm{CH}_{3}\right)_{2} \mathrm{NH}\right)_{2}$}

47

$\begin{array}{lccc}\mathrm{S} & -1.916309 & 1.371320 & -1.106524 \\ \mathrm{O} & -3.338913 & 1.542141 & -1.390451 \\ \mathrm{O} & -1.700257 & 0.554131 & 0.137441 \\ \mathrm{O} & -1.365064 & 0.482382 & -2.288015 \\ \mathrm{O} & -1.140170 & 2.609085 & -1.050570 \\ \mathrm{H} & -0.333045 & 0.425780 & -2.274523 \\ \mathrm{~S} & 1.825392 & -0.821593 & -1.474770 \\ \mathrm{O} & 1.672358 & -0.509857 & 0.020931 \\ \mathrm{O} & 3.259870 & -0.899255 & -1.806980 \\ \mathrm{O} & 1.177633 & 0.316277 & -2.203202 \\ \mathrm{O} & 1.116597 & -2.093730 & -1.751049 \\ \mathrm{~N} & 1.339592 & 2.207425 & 0.202521 \\ \mathrm{C} & 1.354124 & 2.603732 & 1.632382 \\ \mathrm{H} & 2.321804 & 2.335693 & 2.053076 \\ \mathrm{H} & 0.557050 & 2.065386 & 2.143968 \\ \mathrm{H} & 1.180944 & 3.677456 & 1.713965 \\ \mathrm{C} & 2.352948 & 2.897306 & -0.641950 \\ \mathrm{H} & 3.340783 & 2.681235 & -0.240805 \\ \mathrm{H} & 2.151083 & 3.969359 & -0.634493 \\ \mathrm{H} & 2.260737 & 2.497490 & -1.649619\end{array}$




$\begin{array}{lccc}\mathrm{H} & 1.495335 & 1.168038 & 0.141963 \\ \mathrm{H} & 0.399090 & 2.374305 & -0.205856 \\ \mathrm{~N} & -1.216683 & -2.179646 & -0.452526 \\ \mathrm{H} & -1.459474 & -1.190359 & -0.255826 \\ \mathrm{H} & -0.266459 & -2.158473 & -0.926095 \\ \mathrm{C} & -2.201841 & -2.748321 & -1.408307 \\ \mathrm{C} & -1.115099 & -2.899753 & 0.840319 \\ \mathrm{H} & -0.832351 & -3.935707 & 0.651782 \\ \mathrm{H} & -0.341337 & -2.415350 & 1.434106 \\ \mathrm{H} & -2.076509 & -2.850088 & 1.349447 \\ \mathrm{H} & -3.190219 & -2.731738 & -0.954433 \\ \mathrm{H} & -1.900844 & -3.765886 & -1.659436 \\ \mathrm{H} & -2.190934 & -2.128574 & -2.303096 \\ \mathrm{~S} & -4.750959 & -0.277860 & 1.327570 \\ \mathrm{O} & -5.790188 & -0.378882 & 2.300023 \\ \mathrm{O} & -4.182759 & -1.476234 & 0.748717 \\ \mathrm{O} & -3.577461 & 0.570003 & 1.968446 \\ \mathrm{O} & -5.289987 & 0.654649 & 0.171992 \\ \mathrm{H} & -4.555210 & 0.933076 & -0.450285 \\ \mathrm{H} & -2.806617 & 0.596471 & 1.323935 \\ \mathrm{~S} & 4.750967 & -0.240916 & 1.313912 \\ \mathrm{O} & 5.803585 & -0.544364 & 2.232153 \\ \mathrm{O} & 5.219548 & -0.717548 & -0.111541 \\ \mathrm{O} & 4.262957 & 1.122780 & 1.224956 \\ \mathrm{H} & 4.448760 & -0.749632 & -0.770281 \\ \mathrm{O} & 3.533660 & -1.186468 & 1.641477 \\ \mathrm{H} & 2.759516 & -0.966829 & 1.010264\end{array}$

$\left(\mathrm{H}_{2} \mathrm{SO}_{4}\right)_{2} \cdot \mathrm{HSO}_{4}^{-} \cdot\left(\left(\mathrm{CH}_{3}\right)_{2} \mathrm{NH}\right)_{3}$

50

$\begin{array}{lccc}\text { S } & 3.492747 & -1.170698 & 1.020873 \\ \mathrm{O} & 4.081151 & -2.225228 & 1.807348 \\ \mathrm{O} & 2.977450 & -1.597150 & -0.309518 \\ \mathrm{O} & 2.182069 & -0.715116 & 1.850579 \\ \mathrm{O} & 4.322192 & 0.046560 & 0.866212 \\ \mathrm{H} & 1.816842 & 0.137562 & 1.471163 \\ \mathrm{~S} & 0.134837 & 1.520730 & -0.251374 \\ \mathrm{O} & 0.792092 & 1.437089 & -1.590063 \\ \mathrm{O} & -0.732886 & 2.721884 & -0.145970 \\ \mathrm{O} & 1.189867 & 1.528185 & 0.805461 \\ \mathrm{O} & -0.752528 & 0.304619 & -0.051658 \\ \mathrm{~N} & 3.428782 & 1.446352 & -1.254830 \\ \mathrm{C} & 4.126871 & 0.826816 & -2.400261 \\ \mathrm{H} & 3.781757 & 1.277747 & -3.332096 \\ \mathrm{H} & 3.898098 & -0.237646 & -2.384235 \\ \mathrm{H} & 5.203904 & 0.966240 & -2.293552 \\ \mathrm{C} & 3.694620 & 2.885106 & -1.044824 \\ \mathrm{H} & 3.373140 & 3.449386 & -1.921131 \\ \mathrm{H} & 4.759251 & 3.042808 & -0.863879 \\ \mathrm{H} & 3.114879 & 3.197150 & -0.177802 \\ \mathrm{H} & 2.375725 & 1.339210 & -1.376821 \\ \mathrm{H} & 3.709073 & 0.920370 & -0.383936 \\ \mathrm{~N} & 0.282867 & -2.172542 & -0.290026 \\ \mathrm{H} & 1.318807 & -2.032241 & -0.301406 \\ \mathrm{H} & -0.123467 & -1.212686 & -0.132803 \\ \mathrm{C} & -0.094664 & -3.051258 & 0.846831 \\ \mathrm{C} & -0.180653 & -2.651302 & -1.617075 \\ \mathrm{H} & -1.263175 & -2.765299 & -1.581567\end{array}$




$\begin{array}{llll}\mathrm{H} & 0.091291 & -1.901454 & -2.359330 \\ \mathrm{H} & 0.307525 & -3.599425 & -1.848777 \\ \mathrm{H} & 0.327018 & -4.045047 & 0.686830 \\ \mathrm{H} & -1.181715 & -3.092996 & 0.898435 \\ \mathrm{H} & 0.328341 & -2.618970 & 1.752128 \\ \mathrm{~S} & -3.908703 & -1.187417 & -0.535891 \\ \mathrm{O} & -4.162982 & -0.357057 & 0.677946 \\ \mathrm{O} & -5.096983 & -1.454101 & -1.313312 \\ \mathrm{O} & -3.061895 & -2.343499 & -0.230785 \\ \mathrm{O} & -3.016618 & -0.215088 & -1.503340 \\ \mathrm{H} & -2.161242 & -0.022090 & -1.047213 \\ \mathrm{~N} & -3.004773 & 1.960867 & 1.103950 \\ \mathrm{C} & -4.078540 & 2.959647 & 0.933208 \\ \mathrm{C} & -2.498954 & 1.821294 & 2.488263 \\ \mathrm{H} & -2.179160 & 2.217582 & 0.498474 \\ \mathrm{H} & -4.936827 & 2.680501 & 1.545281 \\ \mathrm{H} & -3.716177 & 3.948308 & 1.220052 \\ \mathrm{H} & -4.375682 & 2.967112 & -0.114710 \\ \mathrm{H} & -2.094355 & 2.774337 & 2.830858 \\ \mathrm{H} & -3.308998 & 1.490666 & 3.139182 \\ \mathrm{H} & -1.707094 & 1.075339 & 2.474243 \\ \mathrm{H} & -3.387498 & 1.008361 & 0.811172\end{array}$

$\left(\mathrm{H}_{2} \mathrm{SO}_{4}\right)_{3} \cdot \mathrm{HSO}_{4} \cdot \cdot\left(\left(\mathrm{CH}_{3}\right)_{2} \mathrm{NH}\right)_{3}$

57

$\begin{array}{lccc}\mathrm{S} & -2.115147 & 0.766225 & 1.210535 \\ \mathrm{O} & -3.514156 & 0.847209 & 1.682847 \\ \mathrm{O} & -1.730528 & -0.666687 & 0.946791 \\ \mathrm{O} & -1.970187 & 1.512937 & -0.101306 \\ \mathrm{O} & -1.165034 & 1.350591 & 2.195924 \\ \mathrm{~S} & 2.201823 & 1.105533 & -1.068649 \\ \mathrm{O} & 1.782220 & -0.260066 & -0.520549 \\ \mathrm{O} & 3.591844 & 1.015191 & -1.560442 \\ \mathrm{O} & 2.089200 & 2.086605 & 0.055665 \\ \mathrm{O} & 1.250818 & 1.469435 & -2.154635 \\ \mathrm{~N} & 1.175808 & -0.132844 & 2.169677 \\ \mathrm{C} & 0.968556 & -1.535879 & 2.610683 \\ \mathrm{H} & 1.887027 & -2.093185 & 2.431494 \\ \mathrm{H} & 0.137446 & -1.943715 & 2.039556 \\ \mathrm{H} & 0.717491 & -1.544309 & 3.672178 \\ \mathrm{C} & 2.219796 & 0.596694 & 2.934611 \\ \mathrm{H} & 3.152781 & 0.040921 & 2.866663 \\ \mathrm{H} & 1.896577 & 0.696024 & 3.972040 \\ \mathrm{H} & 2.348947 & 1.572969 & 2.472905 \\ \mathrm{H} & 1.439182 & -0.142359 & 1.155372 \\ \mathrm{H} & 0.270128 & 0.390206 & 2.220748 \\ \mathrm{~N} & -1.013401 & -0.110205 & -2.137473 \\ \mathrm{H} & -1.448287 & 0.275428 & -1.271937 \\ \mathrm{H} & -0.083843 & 0.370471 & -2.189400 \\ \mathrm{C} & -1.856020 & 0.306572 & -3.285471 \\ \mathrm{C} & -0.834754 & -1.580720 & -2.022462 \\ \mathrm{H} & -0.338607 & -1.946723 & -2.922472 \\ \mathrm{H} & -0.212095 & -1.771165 & -1.153919 \\ \mathrm{H} & -1.814041 & -2.038984 & -1.904057 \\ \mathrm{H} & -2.845920 & -0.129019 & -3.164512 \\ \mathrm{H} & -1.391542 & -0.028419 & -4.213592 \\ \mathrm{H} & -1.928437 & 1.393305 & -3.283560 \\ \mathrm{~S} & -4.738971 & -1.764912 & -0.285109\end{array}$




$\begin{array}{lccc}\mathrm{O} & -5.752448 & -2.757772 & -0.466688 \\ \mathrm{O} & -4.104005 & -1.182488 & -1.451130 \\ \mathrm{O} & -3.619871 & -2.383396 & 0.637137 \\ \mathrm{O} & -5.360066 & -0.618490 & 0.597701 \\ \mathrm{H} & -4.639457 & -0.009369 & 0.976560 \\ \mathrm{H} & -2.861549 & -1.711446 & 0.771453 \\ \mathrm{~S} & 4.671078 & -1.881384 & 0.101224 \\ \mathrm{O} & 5.578247 & -2.984782 & 0.033468 \\ \mathrm{O} & 5.343943 & -0.672041 & -0.651483 \\ \mathrm{O} & 4.194621 & -1.438620 & 1.396307 \\ \mathrm{H} & 4.658414 & 0.013918 & -0.951566 \\ \mathrm{O} & 3.421084 & -2.225061 & -0.799557 \\ \mathrm{H} & 2.744284 & -1.467074 & -0.729992 \\ \mathrm{~N} & -0.172613 & 3.505676 & -0.003871 \\ \mathrm{C} & -0.145425 & 4.278828 & -1.266537 \\ \mathrm{C} & -0.396161 & 4.313832 & 1.216541 \\ \mathrm{H} & 0.739847 & 2.967509 & 0.068016 \\ \mathrm{H} & 0.603598 & 5.068622 & -1.190730 \\ \mathrm{H} & 0.135958 & 3.596433 & -2.066957 \\ \mathrm{H} & -1.127624 & 4.717053 & -1.450954 \\ \mathrm{H} & 0.443528 & 4.995129 & 1.363729 \\ \mathrm{H} & -1.320251 & 4.884237 & 1.109242 \\ \mathrm{H} & -0.495896 & 3.627314 & 2.055202 \\ \mathrm{H} & -0.918167 & 2.753957 & -0.052432\end{array}$

$\left(\mathrm{H}_{2} \mathrm{SO}_{4}\right)_{3} \cdot \mathrm{HSO}_{4}^{-} \cdot\left(\left(\mathrm{CH}_{3}\right)_{2} \mathrm{NH}\right)_{4}$

67

$\begin{array}{lccc}\mathrm{S} & 3.152867 & 1.927687 & 0.757211 \\ \mathrm{O} & 1.761259 & 2.315381 & 0.484724 \\ \mathrm{O} & 3.071687 & 0.596114 & 1.687022 \\ \mathrm{O} & 3.808397 & 1.459037 & -0.497607 \\ \mathrm{O} & 3.940466 & 2.862162 & 1.522147 \\ \mathrm{H} & 4.239112 & -0.111526 & -0.541426 \\ \mathrm{~N} & -0.679840 & -1.076988 & 2.272665 \\ \mathrm{H} & 0.078274 & -1.057336 & 1.528534 \\ \mathrm{H} & -1.579363 & -1.301691 & 1.786121 \\ \mathrm{O} & 2.837106 & -2.883820 & 0.093957 \\ \mathrm{O} & 1.556116 & -1.829574 & -1.698853 \\ \mathrm{~S} & 1.528988 & -2.276983 & -0.276065 \\ \mathrm{O} & 1.331532 & -1.019430 & 0.573951 \\ \mathrm{O} & 0.401134 & -3.193131 & 0.006711 \\ \mathrm{H} & -1.143082 & -2.784778 & -0.578060 \\ \mathrm{H} & 2.427026 & -0.039558 & 1.260391 \\ \mathrm{H} & 3.965384 & -1.777432 & -0.213845 \\ \mathrm{~N} & 4.683405 & -1.071640 & -0.562440 \\ \mathrm{C} & 5.844493 & -1.034767 & 0.353259 \\ \mathrm{C} & 4.993723 & -1.422625 & -1.966720 \\ \mathrm{C} & -0.747664 & 0.264154 & 2.902325 \\ \mathrm{C} & -0.367040 & -2.193320 & 3.194422 \\ \mathrm{H} & -0.276401 & -3.100473 & 2.599267 \\ \mathrm{H} & -1.167022 & -2.300553 & 3.928057 \\ \mathrm{H} & 0.579721 & -1.990501 & 3.695979 \\ \mathrm{H} & -1.514901 & 0.267860 & 3.678467 \\ \mathrm{H} & -0.984086 & 0.995147 & 2.131469 \\ \mathrm{H} & 0.223670 & 0.504937 & 3.335241 \\ \mathrm{H} & 5.620965 & -0.647475 & -2.409347 \\ \mathrm{H} & 5.507517 & -2.384538 & -1.996992 \\ \mathrm{H} & 4.047943 & -1.503204 & -2.500924\end{array}$




$\begin{array}{lccc}\mathrm{H} & 6.299408 & -2.024716 & 0.411990 \\ \mathrm{H} & 6.571753 & -0.306895 & -0.008199 \\ \mathrm{H} & 5.485053 & -0.725432 & 1.333426 \\ \mathrm{~S} & -1.574156 & 3.083457 & -0.120618 \\ \mathrm{O} & -1.593170 & 1.572639 & -0.177921 \\ \mathrm{O} & -0.592503 & 3.435480 & 1.098075 \\ \mathrm{O} & -1.016661 & 3.639006 & -1.342262 \\ \mathrm{O} & -2.869904 & 3.594089 & 0.302450 \\ \mathrm{H} & -0.241971 & 0.891178 & -1.226044 \\ \mathrm{~N} & -4.224007 & 0.792188 & -0.207684 \\ \mathrm{H} & -3.203077 & 1.038001 & -0.170498 \\ \mathrm{H} & -4.278565 & -0.229067 & -0.471072 \\ \mathrm{O} & -1.943883 & -2.342028 & -0.978104 \\ \mathrm{O} & -4.282329 & -1.810339 & -0.921645 \\ \mathrm{~S} & -3.286756 & -2.530415 & -0.100943 \\ \mathrm{O} & -3.047111 & -1.789506 & 1.169012 \\ \mathrm{O} & -3.528841 & -3.941065 & 0.079206 \\ \mathrm{H} & 0.323050 & 3.107457 & 0.896124 \\ \mathrm{H} & 0.742736 & -0.348274 & -1.831913 \\ \mathrm{~N} & 0.202147 & 0.516290 & -2.086172 \\ \mathrm{C} & 1.130943 & 1.535584 & -2.647832 \\ \mathrm{C} & -0.891060 & 0.117433 & -3.008859 \\ \mathrm{C} & -4.864170 & 1.614198 & -1.262119 \\ \mathrm{C} & -4.796708 & 0.993178 & 1.146144 \\ \mathrm{H} & -4.336059 & 0.261898 & 1.807080 \\ \mathrm{H} & -5.873625 & 0.821443 & 1.110078 \\ \mathrm{H} & -4.576012 & 2.010513 & 1.466642 \\ \mathrm{H} & -4.714804 & 2.665977 & -1.024862 \\ \mathrm{H} & -5.925334 & 1.367297 & -1.316748 \\ \mathrm{H} & -4.390848 & 1.382147 & -2.215515 \\ \mathrm{H} & -1.478774 & 1.001185 & -3.256200 \\ \mathrm{H} & -0.452460 & -0.308728 & -3.911528 \\ \mathrm{H} & -1.506702 & -0.632634 & -2.513992 \\ \mathrm{H} & 1.544555 & 1.148435 & -3.580667 \\ \mathrm{H} & 0.574263 & 2.455890 & -2.814428 \\ \mathrm{H} & 1.924173 & 1.713454 & -1.925188\end{array}$

\section{Positively charged ammonia-sulfuric acid clusters}

\section{$\mathrm{H}_{2} \mathrm{SO}_{4} \cdot \mathrm{NH}_{4}^{+}$}

12

$\begin{array}{lrrc}\mathrm{H} & 3.753482 & -0.087396 & 0.945444 \\ \mathrm{~S} & -0.681440 & -0.025187 & 0.123569 \\ \mathrm{O} & -1.375673 & -0.943847 & -0.969993 \\ \mathrm{O} & -1.461063 & -0.014165 & 1.314451 \\ \mathrm{O} & -0.675779 & 1.391217 & -0.590636 \\ \mathrm{O} & 0.711939 & -0.393562 & 0.136213 \\ \mathrm{H} & -2.160364 & -1.372627 & -0.589747 \\ \mathrm{H} & -1.542683 & 1.823734 & -0.510432 \\ \mathrm{~N} & 3.366374 & -0.032204 & 0.000108 \\ \mathrm{H} & 2.315005 & -0.097422 & 0.043749 \\ \mathrm{H} & 3.731086 & -0.807956 & -0.558330 \\ \mathrm{H} & 3.646501 & 0.852946 & -0.428815\end{array}$

$\left(\mathrm{H}_{2} \mathrm{SO}_{4}\right)_{2} \cdot \mathrm{NH}_{4}{ }^{+}$ 
19

$\begin{array}{lrrr}\mathrm{S} & 1.919215 & -0.272124 & -0.052576 \\ \mathrm{O} & 1.856012 & 1.147228 & 0.168730 \\ \mathrm{O} & 1.623659 & -1.055378 & 1.278711 \\ \mathrm{O} & 1.135895 & -0.833155 & -1.128114 \\ \mathrm{H} & 0.832504 & 2.602849 & 0.024990 \\ \mathrm{H} & 0.646815 & -1.102509 & 1.410423 \\ \mathrm{~S} & -1.919373 & -0.271765 & 0.052571 \\ \mathrm{O} & -1.624186 & -1.055423 & -1.278544 \\ \mathrm{O} & -1.136794 & -0.833287 & 1.128411 \\ \mathrm{O} & -3.439069 & -0.598578 & 0.231815 \\ \mathrm{O} & -1.855041 & 1.147509 & -0.168914 \\ \mathrm{H} & -0.647381 & -1.103170 & -1.410331 \\ \mathrm{H} & -3.577255 & -1.524024 & 0.497527 \\ \mathrm{~N} & 0.001063 & 3.224780 & -0.000005 \\ \mathrm{H} & -0.830356 & 2.602884 & -0.025335 \\ \mathrm{O} & 3.438604 & -0.600142 & -0.232088 \\ \mathrm{H} & 3.576053 & -1.525769 & -0.497565 \\ \mathrm{H} & -0.021527 & 3.813895 & 0.834099 \\ \mathrm{H} & 0.023609 & 3.814404 & -0.833750\end{array}$

$\mathrm{NH}_{3} \cdot \mathrm{NH}_{4}^{+}$

9

$\begin{array}{lccc}\mathrm{N} & 1.345451 & -0.000039 & -0.000025 \\ \mathrm{H} & 1.733292 & 0.887206 & 0.319069 \\ \mathrm{H} & 1.733813 & -0.719610 & 0.608825 \\ \mathrm{H} & 1.734374 & -0.167164 & -0.927515 \\ \mathrm{~N} & -1.335672 & -0.000009 & -0.000012 \\ \mathrm{H} & -1.695304 & 0.862191 & -0.413384 \\ \mathrm{H} & -0.184341 & -0.000001 & -0.000189 \\ \mathrm{H} & -1.695034 & -0.073121 & 0.953416 \\ \mathrm{H} & -1.695250 & -0.789161 & -0.539964\end{array}$

$\mathrm{H}_{2} \mathrm{SO}_{4} \cdot \mathrm{NH}_{3} \cdot \mathrm{NH}_{4}{ }^{+}$

16

$\begin{array}{lrrr}\mathrm{S} & 0.001296 & -0.460925 & -0.105845 \\ \mathrm{O} & 0.000304 & -1.455865 & 1.166249 \\ \mathrm{O} & 0.003023 & 0.865666 & 0.536280 \\ \mathrm{O} & -1.229063 & -0.729758 & -0.856184 \\ \mathrm{O} & 1.229183 & -0.733662 & -0.857889 \\ \mathrm{H} & 0.001320 & -2.373630 & 0.851157 \\ \mathrm{~N} & -2.800901 & 1.193797 & 0.042578 \\ \mathrm{H} & -3.534741 & 0.922798 & 0.697384 \\ \mathrm{H} & -1.993453 & 1.587236 & 0.546855 \\ \mathrm{H} & -2.382307 & 0.340514 & -0.443148 \\ \mathrm{H} & -3.166985 & 1.864937 & -0.632579 \\ \mathrm{~N} & 2.796522 & 1.196962 & 0.042791 \\ \mathrm{H} & 1.979291 & 1.577990 & 0.542253 \\ \mathrm{H} & 2.391515 & 0.341127 & -0.446697 \\ \mathrm{H} & 3.158352 & 1.874072 & -0.628670 \\ \mathrm{H} & 3.529346 & 0.933403 & 0.701736\end{array}$

$\left(\mathrm{H}_{2} \mathrm{SO}_{4}\right)_{2} \cdot \mathrm{NH}_{3} \cdot \mathrm{NH}_{4}^{+}$ 


$\begin{array}{cccc}\mathrm{S} & -2.120757 & -0.163714 & 0.086989 \\ \mathrm{O} & -1.952396 & 0.870395 & -0.902390 \\ \mathrm{O} & -1.812977 & -1.522725 & -0.297389 \\ \mathrm{~N} & -0.206934 & 3.054690 & -0.925621 \\ \mathrm{H} & -0.391477 & 3.823568 & -0.280860 \\ \mathrm{H} & -1.011193 & 2.399093 & -0.940985 \\ \mathrm{~N} & 0.647109 & -2.795720 & -1.041876 \\ \mathrm{H} & 0.585857 & -3.317019 & -1.916256 \\ \mathrm{H} & -0.275688 & -2.393176 & -0.797482 \\ \mathrm{~S} & 2.021247 & 0.106694 & 0.181573 \\ \mathrm{O} & 3.326991 & -0.047274 & 1.090753 \\ \mathrm{O} & 1.807002 & 1.542230 & -0.008413 \\ \mathrm{O} & 0.997097 & -0.559893 & 1.003847 \\ \mathrm{O} & 2.251920 & -0.620957 & -1.071310 \\ \mathrm{H} & 4.076807 & 0.394295 & 0.662223 \\ \mathrm{O} & -3.622875 & -0.054058 & 0.545826 \\ \mathrm{H} & -3.856119 & -0.774126 & 1.154983 \\ \mathrm{H} & -0.043667 & 3.427815 & -1.860905 \\ \mathrm{H} & 0.633512 & 2.501608 & -0.599110 \\ \mathrm{H} & 0.945714 & -3.419402 & -0.291516 \\ \mathrm{H} & 1.341971 & -1.997802 & -1.124234 \\ \mathrm{O} & -1.382331 & 0.206974 & 1.405325 \\ \mathrm{H} & -0.394228 & -0.062855 & 1.339634\end{array}$

$\left(\mathrm{H}_{2} \mathrm{SO}_{4}\right)_{3} \cdot \mathrm{NH}_{3} \cdot \mathrm{NH}_{4}{ }^{+}$

30

$\begin{array}{lccc}\mathrm{S} & -2.552032 & -0.903454 & -0.008739 \\ \mathrm{O} & -2.576557 & 0.500927 & -0.315567 \\ \mathrm{~N} & -2.062501 & 3.225506 & -0.965903 \\ \mathrm{H} & -2.831476 & 3.839250 & -0.694583 \\ \mathrm{H} & -2.300940 & 2.237804 & -0.761042 \\ \mathrm{~N} & 0.348640 & -1.239703 & 2.763788 \\ \mathrm{H} & 0.581754 & -0.240897 & 2.710492 \\ \mathrm{H} & 0.977727 & -1.740798 & 2.089077 \\ \mathrm{~S} & 2.000137 & -1.486452 & -0.458467 \\ \mathrm{O} & 2.504471 & -2.433645 & -1.637448 \\ \mathrm{O} & 0.692272 & -1.009258 & -1.010950 \\ \mathrm{O} & 2.939773 & -0.386325 & -0.312592 \\ \mathrm{O} & 1.825986 & -2.315111 & 0.729061 \\ \mathrm{H} & 3.359955 & -2.825177 & -1.399073 \\ \mathrm{O} & -1.797836 & -1.716022 & -1.103541 \\ \mathrm{H} & -0.797491 & -1.571389 & -1.061258 \\ \mathrm{H} & -1.880634 & 3.322097 & -1.966102 \\ \mathrm{H} & -1.192292 & 3.470334 & -0.441397 \\ \mathrm{H} & 0.455611 & -1.597876 & 3.711359 \\ \mathrm{H} & -0.621066 & -1.346575 & 2.428732 \\ \mathrm{O} & -2.114428 & -1.308228 & 1.305754 \\ \mathrm{~S} & 0.934468 & 2.129748 & 0.327612 \\ \mathrm{O} & 0.385713 & 1.541511 & -1.026961 \\ \mathrm{O} & 2.474081 & 2.266933 & 0.110258 \\ \mathrm{O} & 0.381937 & 3.456974 & 0.398664 \\ \mathrm{O} & 0.665514 & 1.199900 & 1.397393 \\ \mathrm{H} & 0.543504 & 0.541773 & -1.093541 \\ \mathrm{H} & 2.856038 & 1.357194 & -0.008938 \\ \mathrm{O} & -4.024451 & -1.403851 & -0.266407 \\ \mathrm{H} & -4.126625 & -2.334278 & -0.006728\end{array}$


13

$\begin{array}{lrrr}\mathrm{N} & -2.353209 & -0.505375 & 0.000000 \\ \mathrm{H} & -2.380654 & -1.195865 & 0.747972 \\ \mathrm{H} & -3.182937 & 0.073123 & 0.119282 \\ \mathrm{H} & -2.491233 & -1.021174 & -0.867153 \\ \mathrm{~N} & -0.000003 & 1.034017 & 0.000000 \\ \mathrm{H} & 0.892603 & 0.428764 & -0.000132 \\ \mathrm{H} & -0.892622 & 0.428784 & 0.000127 \\ \mathrm{H} & 0.000197 & 1.633687 & 0.825439 \\ \mathrm{H} & -0.000187 & 1.633697 & -0.825431 \\ \mathrm{~N} & 2.353206 & -0.505378 & 0.000001 \\ \mathrm{H} & 3.182886 & 0.073144 & -0.119514 \\ \mathrm{H} & 2.380594 & -1.196013 & -0.747839 \\ \mathrm{H} & 2.491394 & -1.020997 & 0.867238\end{array}$

\section{$\mathrm{H}_{2} \mathrm{SO}_{4} \cdot\left(\mathrm{NH}_{3}\right)_{2} \cdot \mathrm{NH}_{4}{ }^{+}$}

20

$\begin{array}{lrrc}\mathrm{S} & -0.009115 & -0.004203 & 0.099276 \\ \mathrm{O} & 0.214348 & 1.208862 & 0.911165 \\ \mathrm{O} & 1.064800 & -0.038573 & -1.026342 \\ \mathrm{O} & 0.115789 & -1.244126 & 0.892421 \\ \mathrm{O} & -1.314344 & 0.052899 & -0.612105 \\ \mathrm{~N} & -1.638400 & 2.758208 & -0.083680 \\ \mathrm{H} & -0.808407 & 2.352016 & 0.464307 \\ \mathrm{H} & -2.373219 & 3.095869 & 0.536004 \\ \mathrm{H} & -1.949190 & 1.923186 & -0.610125 \\ \mathrm{H} & -1.344237 & 3.499920 & -0.717431 \\ \mathrm{~N} & 3.479768 & -0.110970 & -0.086355 \\ \mathrm{H} & 2.050751 & -0.068840 & -0.629950 \\ \mathrm{H} & 3.658311 & 0.685083 & 0.521120 \\ \mathrm{H} & 4.180583 & -0.097249 & -0.823131 \\ \mathrm{H} & 3.632937 & -0.951119 & 0.466123 \\ \mathrm{~N} & -1.866086 & -2.626182 & -0.084729 \\ \mathrm{H} & -1.634441 & -3.388312 & -0.720223 \\ \mathrm{H} & -2.614823 & -2.909555 & 0.545398 \\ \mathrm{H} & -0.999323 & -2.279146 & 0.451938 \\ \mathrm{H} & -2.124822 & -1.774502 & -0.610209\end{array}$

$\left(\mathrm{H}_{2} \mathrm{SO}_{4}\right)_{2} \cdot\left(\mathrm{NH}_{3}\right)_{2} \cdot \mathrm{NH}_{4}{ }^{+}$

27

$\begin{array}{lccc}\mathrm{S} & 2.202481 & -0.026478 & 0.001099 \\ \mathrm{O} & 1.560829 & 0.440145 & -1.233462 \\ \mathrm{~N} & -0.013134 & -2.328094 & -1.377575 \\ \mathrm{H} & 0.904099 & -2.147965 & -0.902575 \\ \mathrm{H} & -0.739716 & -2.280953 & -0.640379 \\ \mathrm{O} & 2.034699 & -1.467053 & 0.217829 \\ \mathrm{O} & 1.834018 & 0.773252 & 1.180012 \\ \mathrm{~N} & -0.077755 & 2.569861 & -1.101384 \\ \mathrm{H} & 0.356263 & 3.438630 & -0.793310 \\ \mathrm{H} & 0.647667 & 1.805408 & -1.195630 \\ \mathrm{~N} & -0.191669 & -0.112973 & 2.761997 \\ \mathrm{H} & 0.054540 & -0.646247 & 3.594228 \\ \mathrm{H} & 0.669375 & 0.209545 & 2.245139\end{array}$




$\begin{array}{lrrr}\mathrm{S} & -2.139270 & -0.006976 & -0.069571 \\ \mathrm{O} & -3.718970 & -0.245559 & 0.092732 \\ \mathrm{O} & -1.816381 & 0.032394 & -1.488872 \\ \mathrm{O} & -1.586608 & -1.189162 & 0.628982 \\ \mathrm{O} & -1.806084 & 1.245070 & 0.634254 \\ \mathrm{H} & -4.203153 & 0.423009 & -0.415389 \\ \mathrm{O} & 3.755372 & 0.250685 & -0.287010 \\ \mathrm{H} & 4.288014 & -0.083816 & 0.450703 \\ \mathrm{H} & -0.781065 & 2.232903 & -0.404596 \\ \mathrm{H} & -0.539605 & 2.709449 & -1.999252 \\ \mathrm{H} & -0.212639 & -1.546341 & -2.009216 \\ \mathrm{H} & -0.023868 & -3.216116 & -1.875699 \\ \mathrm{H} & -0.755940 & -0.683655 & 2.091961 \\ \mathrm{H} & -0.752446 & 0.701664 & 3.012582\end{array}$

\section{$\left(\mathrm{H}_{2} \mathrm{SO}_{4}\right)_{3} \cdot\left(\mathrm{NH}_{3}\right)_{2} \cdot \mathrm{NH}_{4}{ }^{+}$}

34

$\begin{array}{lccc}\mathrm{S} & -2.680907 & -1.374538 & -0.001954 \\ \mathrm{O} & -2.735249 & -0.809184 & 1.356394 \\ \mathrm{O} & -3.874549 & -2.441317 & -0.121617 \\ \mathrm{O} & -3.021668 & -0.399657 & -1.043434 \\ \mathrm{H} & -3.721289 & -3.169645 & 0.499328 \\ \mathrm{~S} & 2.971559 & -1.134350 & 0.067211 \\ \mathrm{O} & 3.565798 & 0.302541 & 0.055299 \\ \mathrm{O} & 4.198795 & -1.963414 & 0.614959 \\ \mathrm{O} & 1.932857 & -1.242224 & 1.055855 \\ \mathrm{H} & 4.795193 & -2.205344 & -0.111713 \\ \mathrm{~S} & 0.422016 & 2.284797 & -0.132646 \\ \mathrm{O} & -0.711940 & 2.042420 & 0.783499 \\ \mathrm{O} & 0.273899 & 1.642976 & -1.433468 \\ \mathrm{O} & 1.707903 & 2.027744 & 0.521518 \\ \mathrm{H} & -2.386223 & 2.200208 & 0.339047 \\ \mathrm{O} & -1.422294 & -2.081452 & -0.260811 \\ \mathrm{~N} & 0.064557 & -1.039191 & -2.316378 \\ \mathrm{~N} & -3.373048 & 2.021186 & 0.043480 \\ \mathrm{~N} & -0.302252 & -0.185532 & 2.481244 \\ \mathrm{H} & -3.972287 & 1.952263 & 0.865227 \\ \mathrm{H} & -3.707917 & 2.765077 & -0.566902 \\ \mathrm{H} & -3.364310 & 1.086449 & -0.456935 \\ \mathrm{H} & 2.847243 & 1.001488 & 0.201707 \\ \mathrm{H} & -1.210150 & -0.600625 & 2.164452 \\ \mathrm{H} & -0.228335 & -0.187442 & 3.496979 \\ \mathrm{H} & 0.489600 & -0.698636 & 2.064922 \\ \mathrm{H} & -0.288957 & 0.780069 & 2.107944 \\ \mathrm{H} & -0.553183 & -1.521947 & -1.619845 \\ \mathrm{H} & 1.030667 & -1.374237 & -2.187858 \\ \mathrm{H} & 0.052799 & -0.024009 & -2.106810 \\ \mathrm{H} & -0.262039 & -1.212908 & -3.265083 \\ \mathrm{O} & 2.686117 & -1.568542 & -1.280511 \\ \mathrm{O} & 0.311369 & 3.870557 & -0.361722 \\ \mathrm{H} & 1.063398 & 4.175863 & -0.892377 \\ & & & \\ & & & \end{array}$

$\left(\mathrm{H}_{2} \mathrm{SO}_{4}\right)_{4} \cdot\left(\mathrm{NH}_{3}\right)_{2} \cdot \mathrm{NH}_{4}^{+}$

41

$\begin{array}{lllr}\mathrm{S} & 1.466045 & 2.323763 & -0.039027 \\ \mathrm{O} & 1.364756 & 2.108774 & 1.403007\end{array}$




$\begin{array}{lrrr}\mathrm{O} & 2.592611 & 3.441038 & -0.293539 \\ \mathrm{O} & 2.066149 & 1.168708 & -0.754295 \\ \mathrm{H} & 2.342489 & 4.267378 & 0.146874 \\ \mathrm{~S} & -4.786312 & -0.153956 & -0.492780 \\ \mathrm{O} & -4.514489 & 1.251703 & -0.381657 \\ \mathrm{O} & -3.942397 & -0.792136 & -1.654132 \\ \mathrm{O} & -4.676530 & -0.985103 & 0.683914 \\ \mathrm{H} & -3.002061 & -0.910220 & -1.321746 \\ \mathrm{~S} & -1.244961 & -1.240035 & 0.828906 \\ \mathrm{O} & 0.069758 & -1.847190 & 0.976854 \\ \mathrm{O} & -1.526566 & -0.924907 & -0.609951 \\ \mathrm{O} & -1.469647 & -0.077188 & 1.688138 \\ \mathrm{O} & -2.272167 & -2.369758 & 1.255029 \\ \mathrm{H} & -3.182253 & -2.000653 & 1.236105 \\ \mathrm{O} & 0.225462 & 2.782180 & -0.652318 \\ \mathrm{~N} & 1.862230 & -0.418837 & 2.545233 \\ \mathrm{~N} & -2.154115 & 2.417298 & 0.763663 \\ \mathrm{~N} & 0.844719 & -0.943186 & -2.074334 \\ \mathrm{H} & -1.909251 & 1.510738 & 1.222182 \\ \mathrm{H} & -3.005254 & 2.254650 & 0.207834 \\ \mathrm{H} & -2.329789 & 3.134319 & 1.465250 \\ \mathrm{H} & -1.344937 & 2.686172 & 0.167117 \\ \mathrm{H} & 0.725704 & -1.001308 & -3.084288 \\ \mathrm{H} & -0.061631 & -1.101352 & -1.582612 \\ \mathrm{H} & 1.560338 & -1.613758 & -1.764540 \\ \mathrm{H} & 1.194221 & -0.005051 & -1.795250 \\ \mathrm{H} & 1.140313 & -1.030808 & 2.106551 \\ \mathrm{H} & 1.691854 & 0.557856 & 2.228913 \\ \mathrm{H} & 2.784835 & -0.722788 & 2.199455 \\ \mathrm{H} & 1.817848 & -0.480117 & 3.560753 \\ \mathrm{~S} & 4.376352 & -1.150043 & -0.379848 \\ \mathrm{O} & 4.568521 & 0.337469 & -0.840869 \\ \mathrm{O} & 5.842664 & -1.686937 & -0.586504 \\ \mathrm{O} & 4.135965 & -1.222037 & 1.033931 \\ \mathrm{O} & 3.468287 & -1.822995 & -1.279296 \\ \mathrm{H} & 6.027381 & -1.827887 & -1.529085 \\ \mathrm{H} & 3.679163 & 0.799547 & -0.829785 \\ \mathrm{O} & -6.232758 & -0.263098 & -1.105806 \\ \mathrm{H} & -6.573754 & -1.167494 & -1.011727 \\ & & & \\ & & & \end{array}$

$\left(\mathrm{H}_{2} \mathrm{SO}_{4}\right)_{2} \cdot\left(\mathrm{NH}_{3}\right)_{3} \cdot \mathrm{NH}_{4}^{+}$

31

$\begin{array}{lrrr}\mathrm{S} & 1.836243 & 0.241906 & -0.181308 \\ \mathrm{O} & 0.961415 & 0.627206 & -1.313922 \\ \mathrm{~N} & -0.189803 & -2.109694 & -1.546787 \\ \mathrm{H} & 0.719948 & -1.934475 & -1.044417 \\ \mathrm{H} & -0.934417 & -2.139156 & -0.824954 \\ \mathrm{O} & 1.875925 & -1.226271 & -0.014546 \\ \mathrm{O} & 1.416453 & 0.927798 & 1.062323 \\ \mathrm{~N} & -0.705876 & 2.638222 & -0.812602 \\ \mathrm{H} & -0.246502 & 3.421647 & -0.350541 \\ \mathrm{H} & 0.014326 & 1.893172 & -1.070573 \\ \mathrm{~N} & -0.437610 & -0.196130 & 2.637884 \\ \mathrm{H} & -0.117367 & -0.660533 & 3.485036 \\ \mathrm{H} & 0.372012 & 0.215043 & 2.083439 \\ \mathrm{~S} & -2.587535 & -0.172053 & 0.006219 \\ \mathrm{O} & -4.121025 & -0.580102 & 0.266759 \\ \mathrm{O} & -2.391206 & 0.022095 & -1.419930\end{array}$




$\begin{array}{lrrr}\mathrm{O} & -1.871754 & -1.346383 & 0.553844 \\ \mathrm{O} & -2.317556 & 1.042792 & 0.802744 \\ \mathrm{H} & -4.702486 & 0.066952 & -0.160141 \\ \mathrm{O} & 3.253482 & 0.740310 & -0.555533 \\ \mathrm{H} & 4.036538 & 0.184643 & -0.106073 \\ \mathrm{H} & -1.389413 & 2.180866 & -0.168605 \\ \mathrm{H} & -1.189186 & 2.962011 & -1.648577 \\ \mathrm{H} & -0.379556 & -1.282732 & -2.119889 \\ \mathrm{H} & -0.169756 & -2.954565 & -2.113971 \\ \mathrm{H} & -0.947804 & -0.848778 & 2.005224 \\ \mathrm{H} & -1.094964 & 0.552636 & 2.859385 \\ \mathrm{~N} & 5.210706 & -0.614074 & 0.457412 \\ \mathrm{H} & 4.934160 & -1.589554 & 0.537670 \\ \mathrm{H} & 5.499349 & -0.299336 & 1.380076 \\ \mathrm{H} & 6.028007 & -0.573320 & -0.146932\end{array}$

\section{$\left(\mathrm{H}_{2} \mathrm{SO}_{4}\right)_{3} \cdot\left(\mathrm{NH}_{3}\right)_{3} \cdot \mathrm{NH}_{4}{ }^{+}$}

38

$\begin{array}{cccc}\mathrm{S} & 2.206788 & -1.858397 & -0.044660 \\ \mathrm{O} & 1.056851 & -1.251929 & -0.756932 \\ \mathrm{O} & 2.247222 & -3.311476 & -0.760452 \\ \mathrm{O} & 3.478354 & -1.207395 & -0.332600 \\ \mathrm{H} & 2.964508 & -3.835353 & -0.372500 \\ \mathrm{~S} & -3.144821 & -0.490856 & 0.206796 \\ \mathrm{O} & -3.192381 & 0.821634 & 0.852719 \\ \mathrm{O} & -4.637569 & -1.099046 & 0.244278 \\ \mathrm{O} & -2.863170 & -0.437403 & -1.232900 \\ \mathrm{H} & -4.967364 & -1.076044 & 1.155238 \\ \mathrm{~S} & 0.947881 & 2.351266 & -0.039446 \\ \mathrm{O} & 1.225256 & 1.586273 & 1.187932 \\ \mathrm{O} & 2.192194 & 2.738871 & -0.712227 \\ \mathrm{O} & -0.022578 & 3.427772 & 0.095132 \\ \mathrm{O} & 0.169221 & 1.323972 & -1.029929 \\ \mathrm{H} & 0.612998 & 0.445700 & -1.010401 \\ \mathrm{O} & 1.948541 & -2.086762 & 1.371738 \\ \mathrm{~N} & -0.104211 & -0.488538 & 2.407834 \\ \mathrm{~N} & -2.431110 & 2.754167 & -0.951481 \\ \mathrm{~N} & -1.186242 & -2.558324 & -1.623935 \\ \mathrm{~N} & 4.322232 & 1.384382 & 0.121473 \\ \mathrm{H} & -1.499192 & -3.195619 & -0.891115 \\ \mathrm{H} & -1.871648 & -1.760141 & -1.651114 \\ \mathrm{H} & -0.252736 & -2.168982 & -1.348781 \\ \mathrm{H} & -1.131707 & -3.043955 & -2.517417 \\ \mathrm{H} & -3.070453 & 3.494037 & -1.234502 \\ \mathrm{H} & -1.533331 & 3.144170 & -0.564183 \\ \mathrm{H} & -2.859020 & 2.134124 & -0.227378 \\ \mathrm{H} & -2.196309 & 2.157799 & -1.746202 \\ \mathrm{H} & 4.059210 & 0.382163 & -0.006471 \\ \mathrm{H} & 4.486641 & 1.570652 & 1.109600 \\ \mathrm{H} & 5.165068 & 1.595818 & -0.410545 \\ \mathrm{H} & 3.506061 & 1.983848 & -0.217381 \\ \mathrm{H} & 0.628079 & -1.193714 & 2.196475 \\ \mathrm{H} & -0.985019 & -0.775891 & 1.933408 \\ \mathrm{H} & 0.241983 & 0.415114 & 2.013619 \\ \mathrm{H} & -0.250953 & -0.406693 & 3.411583 \\ \mathrm{O} & -2.278074 & -1.447143 & 0.915465\end{array}$


45

$\begin{array}{lccc}\mathrm{S} & 1.593979 & 2.217709 & 0.903242 \\ \mathrm{O} & 2.185478 & 3.002106 & -0.187406 \\ \mathrm{O} & 0.519341 & 1.205671 & 0.233919 \\ \mathrm{O} & 2.609480 & 1.364407 & 1.546369 \\ \mathrm{O} & 0.752657 & 2.980415 & 1.813281 \\ \mathrm{H} & 0.985617 & 0.533318 & -0.314291 \\ \mathrm{~S} & 3.040922 & -1.428443 & -1.128166 \\ \mathrm{O} & 1.654354 & -0.919677 & -1.028090 \\ \mathrm{O} & 2.833953 & -2.600115 & -2.229395 \\ \mathrm{O} & 3.973238 & -0.466175 & -1.703342 \\ \mathrm{O} & 3.511013 & -2.066897 & 0.094900 \\ \mathrm{H} & 3.676664 & -3.059600 & -2.363442 \\ \mathrm{~S} & -3.371190 & 0.813976 & -1.670414 \\ \mathrm{O} & -3.122722 & 2.047688 & -0.962831 \\ \mathrm{O} & -2.281986 & 0.150371 & -2.331705 \\ \mathrm{O} & -4.137262 & -0.184071 & -0.748190 \\ \mathrm{~N} & -1.911116 & 2.396762 & 1.652111 \\ \mathrm{H} & -2.488760 & 2.999552 & 2.235218 \\ \mathrm{H} & -1.995726 & 1.412282 & 1.967881 \\ \mathrm{H} & -0.904717 & 2.690212 & 1.709147 \\ \mathrm{H} & -2.239679 & 2.436878 & 0.676795 \\ \mathrm{~N} & 2.171111 & -1.201770 & 2.394303 \\ \mathrm{H} & 2.302606 & -0.174959 & 2.236578 \\ \mathrm{H} & 2.698935 & -1.685740 & 1.641212 \\ \mathrm{H} & 1.167050 & -1.437423 & 2.296144 \\ \mathrm{H} & 2.516099 & -1.477177 & 3.311170 \\ \mathrm{~N} & -0.693978 & -2.147093 & -1.772833 \\ \mathrm{H} & -1.178676 & -1.434840 & -2.334194 \\ \mathrm{H} & -1.259514 & -2.259830 & -0.908112 \\ \mathrm{H} & -0.618233 & -3.024984 & -2.283015 \\ \mathrm{H} & 0.255476 & -1.788595 & -1.521166 \\ \mathrm{~N} & 4.586460 & 2.105383 & -0.905926 \\ \mathrm{H} & 5.022642 & 2.640898 & -1.654824 \\ \mathrm{H} & 4.415826 & 1.122862 & -1.217191 \\ \mathrm{H} & 5.194775 & 2.100578 & -0.088396 \\ \mathrm{H} & 3.647530 & 2.533577 & -0.634125 \\ \mathrm{~S} & -1.859006 & -1.583666 & 1.729800 \\ \mathrm{O} & -2.270453 & -0.317835 & 2.322412 \\ \mathrm{O} & -2.464034 & -2.749266 & 2.653917 \\ \mathrm{H} & -0.440531 & -1.882834 & 1.707065 \\ \mathrm{O} & -3.381721 & -2.537566 & 2.881797 \\ \mathrm{H} & -5.45790351 & 1.088502 & -2.778205 \\ \mathrm{H} & -1.776949 & 0.372860 \\ \mathrm{H} & 1.827576 & -2.510679 \\ \mathrm{H} & -0.805945 & -0.255974\end{array}$

$\left(\mathrm{H}_{2} \mathrm{SO}_{4}\right)_{3} \cdot\left(\mathrm{NH}_{3}\right)_{4} \cdot \mathrm{NH}_{4}{ }^{+}$

42

$\begin{array}{llll}\mathrm{S} & -1.416896 & 1.951286 & -0.110997 \\ \mathrm{O} & -1.817642 & 1.650493 & 1.295280 \\ \mathrm{O} & -2.725835 & 1.615672 & -0.988633 \\ \mathrm{O} & -1.109681 & 3.349090 & -0.340828 \\ \mathrm{O} & -0.366759 & 1.032596 & -0.560235 \\ \mathrm{H} & -2.681526 & 0.652422 & -1.220994\end{array}$




$\begin{array}{lccc}\mathrm{S} & -2.307062 & -1.967028 & -0.174104 \\ \mathrm{O} & -0.907636 & -2.221979 & 0.206645 \\ \mathrm{O} & -2.882415 & -3.352538 & -0.758905 \\ \mathrm{O} & -3.136862 & -1.607009 & 0.983736 \\ \mathrm{O} & -2.451826 & -1.066614 & -1.318642 \\ \mathrm{H} & -2.814760 & -4.034942 & -0.074155 \\ \mathrm{~N} & 1.765383 & 3.107963 & -0.594378 \\ \mathrm{H} & 2.350059 & 3.937065 & -0.677410 \\ \mathrm{H} & 2.012687 & 2.547768 & 0.254556 \\ \mathrm{H} & 1.966701 & 2.436956 & -1.349781 \\ \mathrm{H} & 0.757665 & 3.352778 & -0.582433 \\ \mathrm{~N} & 0.571980 & -1.100331 & -2.038105 \\ \mathrm{H} & -0.183047 & -0.423540 & -1.861232 \\ \mathrm{H} & 0.488709 & -1.801678 & -1.291123 \\ \mathrm{H} & 1.477510 & -0.582182 & -1.930951 \\ \mathrm{H} & 0.471133 & -1.534943 & -2.952256 \\ \mathrm{~N} & 0.184859 & -0.273577 & 2.053341 \\ \mathrm{H} & 1.079980 & 0.155476 & 1.717025 \\ \mathrm{H} & -0.079060 & -1.059622 & 1.431649 \\ \mathrm{H} & 0.289272 & -0.606379 & 3.009425 \\ \mathrm{H} & -0.562719 & 0.440057 & 1.986715 \\ \mathrm{~N} & -4.380293 & 0.765014 & 1.360280 \\ \mathrm{H} & -4.966560 & 0.821379 & 2.190929 \\ \mathrm{H} & -4.090197 & -0.227685 & 1.167549 \\ \mathrm{H} & -4.870107 & 1.152967 & 0.554329 \\ \mathrm{H} & -3.476599 & 1.289366 & 1.479326 \\ \mathrm{~S} & 3.029801 & 0.061252 & 0.019800 \\ \mathrm{O} & 4.571028 & 0.156261 & 0.160367 \\ \mathrm{O} & 2.686302 & 0.528639 & -1.351140 \\ \mathrm{O} & 2.451663 & 1.006023 & 1.028297 \\ \mathrm{O} & 2.595694 & -1.313212 & 0.263109 \\ \mathrm{~N} & 5.632525 & -2.117662 & 0.733589 \\ \mathrm{H} & 4.850757 & -2.764858 & 0.794330 \\ \mathrm{H} & 6.272424 & -2.460223 & 0.023055 \\ \mathrm{H} & 5.021698 & -0.778391 & 0.397527 \\ \mathrm{H} & 6.123056 & -2.129164 & 1.623234\end{array}$

$\left(\mathrm{H}_{2} \mathrm{SO}_{4}\right)_{4} \cdot\left(\mathrm{NH}_{3}\right)_{4} \cdot \mathrm{NH}_{4}{ }^{+}$

49

$\begin{array}{lccc}\mathrm{S} & -1.460159 & 2.029528 & 0.182555 \\ \mathrm{O} & -1.424976 & 1.164689 & 1.401868 \\ \mathrm{O} & -3.007845 & 2.243222 & -0.117809 \\ \mathrm{O} & -0.924314 & 3.360416 & 0.425839 \\ \mathrm{O} & -0.860588 & 1.357982 & -0.977579 \\ \mathrm{H} & -3.375896 & 1.436104 & -0.597309 \\ \mathrm{~S} & -4.367121 & -1.093633 & -0.422808 \\ \mathrm{O} & -3.343211 & -2.111828 & -0.242360 \\ \mathrm{O} & -5.478480 & -1.841243 & -1.308004 \\ \mathrm{O} & -5.044706 & -0.668079 & 0.811914 \\ \mathrm{O} & -3.896285 & 0.055489 & -1.223104 \\ \mathrm{H} & -6.240206 & -1.254198 & -1.424065 \\ \mathrm{~S} & 1.978908 & -2.647644 & 0.227778 \\ \mathrm{O} & 3.388265 & -2.953628 & 0.003911 \\ \mathrm{O} & 1.465390 & -1.546702 & -0.613399 \\ \mathrm{O} & 1.128312 & -3.889093 & -0.365524 \\ \mathrm{~N} & 1.132503 & 3.641270 & -1.405698 \\ \mathrm{H} & 1.155230 & 4.414979 & -2.066490 \\ \mathrm{H} & 2.062211 & 3.512241 & -0.934284\end{array}$




$\begin{array}{cccc}\mathrm{H} & 0.928999 & 2.757909 & -1.881442 \\ \mathrm{H} & 0.378700 & 3.766936 & -0.696865 \\ \mathrm{~N} & -1.123821 & -1.350485 & -1.695157 \\ \mathrm{H} & -1.159206 & -0.323490 & -1.546105 \\ \mathrm{H} & -1.908971 & -1.777757 & -1.151993 \\ \mathrm{H} & -0.201641 & -1.674998 & -1.355911 \\ \mathrm{H} & -1.239330 & -1.567102 & -2.682954 \\ \mathrm{~N} & 1.033240 & 0.101969 & 2.359417 \\ \mathrm{H} & 1.862814 & 0.529300 & 1.882967 \\ \mathrm{H} & 1.099927 & -0.922730 & 2.181448 \\ \mathrm{H} & 1.084061 & 0.285825 & 3.359254 \\ \mathrm{H} & 0.146281 & 0.485688 & 1.976322 \\ \mathrm{~N} & -4.000896 & 1.144469 & 2.454965 \\ \mathrm{H} & -4.163982 & 0.923857 & 3.435588 \\ \mathrm{H} & -4.455423 & 0.413388 & 1.826092 \\ \mathrm{H} & -4.387101 & 2.061721 & 2.232862 \\ \mathrm{H} & -2.979897 & 1.161425 & 2.228788 \\ \mathrm{~S} & 3.591207 & 1.423101 & -0.230209 \\ \mathrm{O} & 4.824937 & 0.926268 & -0.846829 \\ \mathrm{O} & 2.359759 & 0.969940 & -1.185290 \\ \mathrm{O} & 3.459768 & 2.873046 & -0.230501 \\ \mathrm{H} & 2.137858 & 0.022091 & -1.026826 \\ \mathrm{O} & 1.612827 & -2.513807 & 1.636086 \\ \mathrm{O} & 3.346297 & 0.766520 & 1.067117 \\ \mathrm{H} & 1.291340 & -4.675339 & 0.176865 \\ \mathrm{~N} & 5.637048 & -1.390802 & 0.188289 \\ \mathrm{H} & 4.795599 & -2.012174 & 0.149081 \\ \mathrm{H} & 6.406495 & -1.812306 & -0.329595 \\ \mathrm{H} & 5.375384 & -0.447928 & -0.235269 \\ \mathrm{H} & 5.913673 & -1.255548 & 1.159385 \\ & & & \end{array}$

\section{$\left(\mathrm{H}_{2} \mathrm{SO}_{4}\right)_{5} \cdot\left(\mathrm{NH}_{3}\right)_{4} \cdot \mathrm{NH}_{4}{ }^{+}$}

56

$\begin{array}{lccc}\mathrm{S} & 3.311783 & -0.540943 & 0.946250 \\ \mathrm{O} & 3.887567 & -0.216507 & -0.360126 \\ \mathrm{O} & 3.511697 & -2.099700 & 1.212440 \\ \mathrm{O} & 3.992334 & 0.101820 & 2.064012 \\ \mathrm{O} & 1.834873 & -0.322337 & 0.960671 \\ \mathrm{H} & 3.092496 & -2.609606 & 0.478794 \\ \mathrm{~S} & 0.824818 & -3.405575 & -1.453934 \\ \mathrm{O} & 1.823619 & -3.506508 & -0.375501 \\ \mathrm{O} & 0.785306 & -4.843210 & -2.181213 \\ \mathrm{O} & -0.529243 & -3.298493 & -0.903691 \\ \mathrm{O} & 1.152258 & -2.422069 & -2.479192 \\ \mathrm{H} & 1.652102 & -5.022273 & -2.575310 \\ \mathrm{~S} & -3.025477 & -1.148669 & 1.883142 \\ \mathrm{O} & -2.103161 & -0.007475 & 1.973678 \\ \mathrm{O} & -2.359656 & -2.425390 & 2.103422 \\ \mathrm{O} & -4.136643 & -0.969073 & 3.029005 \\ \mathrm{~N} & 4.786154 & 2.591531 & 1.284971 \\ \mathrm{H} & 5.680763 & 2.577104 & 0.797656 \\ \mathrm{H} & 4.013405 & 2.841390 & 0.602308 \\ \mathrm{H} & 4.821051 & 3.270854 & 2.043160 \\ \mathrm{H} & 4.577157 & 1.631930 & 1.664428 \\ \mathrm{~N} & 0.442157 & -2.549331 & 1.974876 \\ \mathrm{H} & 0.832148 & -1.614657 & 1.734195 \\ \mathrm{H} & 0.755189 & -3.187729 & 1.227654 \\ \mathrm{H} & -0.597420 & -2.519163 & 2.012532\end{array}$




$\begin{array}{lccc}\mathrm{H} & 0.822856 & -2.853298 & 2.869236 \\ \mathrm{~N} & -2.885275 & -2.284143 & -1.693921 \\ \mathrm{H} & -2.710918 & -1.328400 & -2.034935 \\ \mathrm{H} & -3.394715 & -2.171752 & -0.800126 \\ \mathrm{H} & -3.426833 & -2.826403 & -2.363603 \\ \mathrm{H} & -1.957759 & -2.742134 & -1.495887 \\ \mathrm{~N} & 2.087818 & 0.234805 & -2.409941 \\ \mathrm{H} & 2.482674 & 0.499358 & -3.309609 \\ \mathrm{H} & 1.448019 & 0.987164 & -2.067157 \\ \mathrm{H} & 1.604392 & -0.677074 & -2.489102 \\ \mathrm{H} & 2.848328 & 0.119682 & -1.704922 \\ \mathrm{~S} & 1.376877 & 3.039982 & -0.265301 \\ \mathrm{O} & 0.864063 & 4.393923 & -0.038307 \\ \mathrm{O} & 1.121273 & 2.317322 & 1.141723 \\ \mathrm{O} & 2.829294 & 2.998204 & -0.501898 \\ \mathrm{H} & 1.313744 & 1.348847 & 1.064915 \\ \mathrm{O} & -3.858426 & -1.112524 & 0.663555 \\ \mathrm{O} & 0.630858 & 2.301770 & -1.290347 \\ \mathrm{H} & -3.720921 & -1.066716 & 3.898772 \\ \mathrm{~N} & -1.611329 & 5.033009 & -0.714652 \\ \mathrm{H} & -2.106377 & 5.308345 & 0.132706 \\ \mathrm{H} & -0.610787 & 4.745121 & -0.458574 \\ \mathrm{H} & -2.113174 & 4.232778 & -1.148060 \\ \mathrm{H} & -1.592064 & 5.818973 & -1.362852 \\ \mathrm{~S} & -2.929470 & 1.617941 & -0.999882 \\ \mathrm{O} & -2.230794 & 2.019552 & 0.343903 \\ \mathrm{O} & -4.385625 & 1.196361 & -0.576932 \\ \mathrm{O} & -2.264739 & 0.486463 & -1.601681 \\ \mathrm{O} & -3.055454 & 2.842500 & -1.743010 \\ \mathrm{H} & -2.119801 & 1.238325 & 0.975474 \\ \mathrm{H} & -4.341928 & 0.337434 & -0.069496\end{array}$

\section{Positively charged ammonia-sulfuric acid clusters}

$$
\left(\mathrm{H}_{2} \mathrm{SO}_{4}\right) \cdot\left(\mathrm{CH}_{3}\right)_{2} \mathrm{NH}_{2}^{+}
$$

18

$\begin{array}{llcc}\mathrm{H} & -2.696159 & 0.014717 & -1.437322 \\ \mathrm{~S} & 1.347121 & 0.028635 & 0.003181 \\ \mathrm{O} & 2.472549 & -0.041593 & -1.109667 \\ \mathrm{O} & 1.108724 & 1.408694 & 0.254558 \\ \mathrm{O} & 2.016112 & -0.678890 & 1.257546 \\ \mathrm{O} & 0.264400 & -0.855871 & -0.363164 \\ \mathrm{H} & 2.594261 & -0.952942 & -1.425941 \\ \mathrm{H} & 2.649337 & -0.078067 & 1.686884 \\ \mathrm{~N} & -2.426603 & -0.043369 & -0.453004 \\ \mathrm{H} & -1.438121 & -0.364919 & -0.442704 \\ \mathrm{C} & -3.281284 & -1.068493 & 0.232870 \\ \mathrm{H} & -4.320280 & -0.745248 & 0.201826 \\ \mathrm{H} & -2.946601 & -1.159330 & 1.264408 \\ \mathrm{H} & -3.165476 & -2.021614 & -0.280124 \\ \mathrm{C} & -2.491092 & 1.328013 & 0.156054 \\ \mathrm{H} & -3.512181 & 1.699058 & 0.089244 \\ \mathrm{H} & -1.807985 & 1.982947 & -0.381402 \\ \mathrm{H} & -2.184546 & 1.254976 & 1.197548\end{array}$


25

$\begin{array}{lccc}\mathrm{S} & -1.978644 & -0.668544 & 0.056018 \\ \mathrm{O} & -1.817112 & 0.744372 & -0.140500 \\ \mathrm{O} & -1.725237 & -1.453297 & -1.284482 \\ \mathrm{O} & -1.247180 & -1.303404 & 1.128834 \\ \mathrm{H} & 0.971555 & 2.075355 & -0.033211 \\ \mathrm{H} & -0.752033 & -1.568636 & -1.405444 \\ \mathrm{~S} & 1.856489 & -0.945064 & -0.058156 \\ \mathrm{O} & 1.481932 & -1.681552 & 1.281136 \\ \mathrm{O} & 1.033131 & -1.454203 & -1.131326 \\ \mathrm{O} & 3.341080 & -1.419381 & -0.223964 \\ \mathrm{O} & 1.922213 & 0.475301 & 0.140593 \\ \mathrm{H} & 0.500990 & -1.657041 & 1.394959 \\ \mathrm{H} & 3.388136 & -2.358928 & -0.470121 \\ \mathrm{~N} & 0.210604 & 2.770173 & -0.005952 \\ \mathrm{H} & -0.645901 & 2.196986 & 0.000976 \\ \mathrm{C} & 0.240670 & 3.602878 & -1.249279 \\ \mathrm{H} & 1.179741 & 4.152417 & -1.284312 \\ \mathrm{H} & -0.598528 & 4.295968 & -1.230555 \\ \mathrm{H} & 0.159965 & 2.944631 & -2.112457 \\ \mathrm{C} & 0.302779 & 3.551010 & 1.267804 \\ \mathrm{H} & 1.236858 & 4.109983 & 1.272650 \\ \mathrm{H} & 0.281370 & 2.855836 & 2.105105 \\ \mathrm{H} & -0.543070 & 4.233769 & 1.326281 \\ \mathrm{O} & -3.519463 & -0.902380 & 0.220601 \\ \mathrm{H} & -3.714446 & -1.820807 & 0.473717\end{array}$

$\left(\mathrm{CH}_{3}\right)_{2} \mathrm{NH} \cdot\left(\mathrm{CH}_{3}\right)_{2} \mathrm{NH}_{2}{ }^{+}$

21

$\begin{array}{lrrr}\mathrm{N} & -1.312253 & 0.000101 & -0.370481 \\ \mathrm{H} & -1.354994 & 0.000601 & -1.387769 \\ \mathrm{C} & -1.987356 & -1.228254 & 0.108635 \\ \mathrm{C} & -1.987816 & 1.227703 & 0.109848 \\ \mathrm{H} & -1.901278 & -1.289642 & 1.195695 \\ \mathrm{H} & -3.050935 & -1.238795 & -0.149874 \\ \mathrm{H} & -1.513198 & -2.106917 & -0.331621 \\ \mathrm{H} & -1.901875 & 1.287937 & 1.196981 \\ \mathrm{H} & -3.051369 & 1.238159 & -0.148780 \\ \mathrm{H} & -1.513885 & 2.106994 & -0.329399 \\ \mathrm{~N} & 1.321622 & 0.000139 & 0.373097 \\ \mathrm{H} & 1.334772 & 0.000621 & 1.394015 \\ \mathrm{H} & 0.248301 & -0.000217 & 0.067510 \\ \mathrm{C} & 1.976299 & -1.250728 & -0.116472 \\ \mathrm{C} & 1.975360 & 1.251048 & -0.117602 \\ \mathrm{H} & 3.022073 & 1.266176 & 0.184361 \\ \mathrm{H} & 1.457658 & 2.112242 & 0.302279 \\ \mathrm{H} & 1.908486 & 1.276178 & -1.204502 \\ \mathrm{H} & 3.023004 & -1.264823 & 0.185573 \\ \mathrm{H} & 1.459212 & -2.111942 & 0.304118 \\ \mathrm{H} & 1.909526 & -1.276857 & -1.203355\end{array}$

$\mathrm{H}_{2} \mathrm{SO}_{4} \cdot\left(\mathrm{CH}_{3}\right)_{2} \mathrm{NH} \cdot\left(\mathrm{CH}_{3}\right)_{2} \mathrm{NH}_{2}{ }^{+}$ 


$\begin{array}{lccc}\mathrm{S} & 0.093795 & -0.181845 & -0.001433 \\ \mathrm{O} & -0.902850 & 0.888078 & -0.001331 \\ \mathrm{O} & 0.900018 & -0.275985 & -1.217770 \\ \mathrm{O} & 0.900099 & -0.275581 & 1.215024 \\ \mathrm{~N} & 3.354843 & 0.174368 & -0.000146 \\ \mathrm{H} & 2.706317 & 0.020580 & -0.795594 \\ \mathrm{H} & 2.702441 & 0.021126 & 0.792521 \\ \mathrm{C} & 4.436799 & -0.851108 & 0.002902 \\ \mathrm{C} & 3.832061 & 1.588251 & 0.000420 \\ \mathrm{H} & 5.051483 & -0.725747 & -0.887220 \\ \mathrm{H} & 5.046889 & -0.725049 & 0.896074 \\ \mathrm{H} & 3.981451 & -1.839871 & 0.002139 \\ \mathrm{H} & 4.433823 & 1.762142 & -0.890327 \\ \mathrm{H} & 4.429601 & 1.762755 & 0.893879 \\ \mathrm{H} & 2.965886 & 2.247498 & -0.001843 \\ \mathrm{~N} & -3.428898 & 0.008308 & 0.000773 \\ \mathrm{H} & -3.186754 & -0.985657 & -0.000102 \\ \mathrm{H} & -2.481561 & 0.474110 & 0.000026 \\ \mathrm{C} & -4.154696 & 0.351238 & 1.259267 \\ \mathrm{C} & -4.157473 & 0.352876 & -1.255660 \\ \mathrm{H} & -5.106142 & -0.181326 & -1.286089 \\ \mathrm{H} & -3.538394 & 0.070449 & -2.105390 \\ \mathrm{H} & -4.334172 & 1.427019 & -1.272071 \\ \mathrm{H} & -5.103271 & -0.183048 & 1.291096 \\ \mathrm{H} & -3.533719 & 0.067723 & 2.107248 \\ \mathrm{H} & -4.331393 & 1.425351 & 1.277454 \\ \mathrm{O} & -0.904835 & -1.504943 & -0.001159 \\ \mathrm{H} & -0.364410 & -2.307345 & -0.002957\end{array}$

$\left(\mathrm{H}_{2} \mathrm{SO}_{4}\right)_{2} \cdot\left(\mathrm{CH}_{3}\right)_{2} \mathrm{NH} \cdot\left(\mathrm{CH}_{3}\right)_{2} \mathrm{NH}_{2}{ }^{+}$

35

$\begin{array}{lccc}\mathrm{S} & 0.638213 & 2.087620 & -0.224616 \\ \mathrm{C} & -3.951129 & 0.400821 & -0.497005 \\ \mathrm{H} & -4.736007 & -0.321515 & -0.278288 \\ \mathrm{H} & -4.385016 & 1.386511 & -0.658489 \\ \mathrm{H} & -3.386022 & 0.084772 & -1.371255 \\ \mathrm{O} & -0.770027 & 2.246891 & 0.057359 \\ \mathrm{O} & 1.522234 & 1.734904 & 0.854946 \\ \mathrm{~N} & -3.011997 & 0.460799 & 0.663300 \\ \mathrm{C} & -3.639993 & 0.874025 & 1.951867 \\ \mathrm{H} & -2.560412 & -0.473840 & 0.772028 \\ \mathrm{H} & -2.240488 & 1.104570 & 0.434766 \\ \mathrm{H} & -4.071127 & 1.867693 & 1.839611 \\ \mathrm{H} & -2.874939 & 0.887434 & 2.726269 \\ \mathrm{H} & -4.417346 & 0.157913 & 2.213557 \\ \mathrm{C} & 3.931299 & -0.691137 & -0.529606 \\ \mathrm{H} & 3.392243 & -0.275693 & -1.379064 \\ \mathrm{H} & 4.239323 & -1.712328 & -0.747661 \\ \mathrm{H} & 4.802019 & -0.077440 & -0.303415 \\ \mathrm{~N} & 3.015773 & -0.702279 & 0.650120 \\ \mathrm{C} & 3.622946 & -1.234862 & 1.905206 \\ \mathrm{H} & 2.168621 & -1.261899 & 0.416113 \\ \mathrm{H} & 2.657229 & 0.251670 & 0.811320 \\ \mathrm{H} & 2.874343 & -1.203903 & 2.694857 \\ \mathrm{H} & 3.937667 & -2.263398 & 1.736676 \\ \mathrm{H} & 4.480247 & -0.621811 & 2.179314 \\ \mathrm{~S} & -0.647213 & -1.915341 & -0.341326 \\ \mathrm{O} & -1.030536 & -3.094670 & -1.360456\end{array}$




$\begin{array}{lrrr}\mathrm{O} & -0.832842 & -0.719388 & -1.194514 \\ \mathrm{O} & 0.738099 & -2.130735 & 0.069391 \\ \mathrm{O} & -1.610187 & -1.946111 & 0.757320 \\ \mathrm{H} & -1.022531 & -3.940053 & -0.885599 \\ \mathrm{O} & 0.837384 & 1.119233 & -1.427435 \\ \mathrm{H} & 0.162183 & 0.326211 & -1.381826 \\ \mathrm{O} & 1.229713 & 3.431666 & -0.811599 \\ \mathrm{H} & 0.648134 & 3.791612 & -1.500647\end{array}$

$\left(\left(\mathrm{CH}_{3}\right)_{2} \mathrm{NH}\right)_{2} \cdot\left(\mathrm{CH}_{3}\right)_{2} \mathrm{NH}_{2}{ }^{+}$

31

$\begin{array}{lccc}\mathrm{N} & -2.279242 & -0.665080 & -0.355486 \\ \mathrm{H} & -2.213356 & -1.042365 & -1.298274 \\ \mathrm{C} & -2.287234 & -1.804189 & 0.584222 \\ \mathrm{C} & -3.524957 & 0.124789 & -0.274813 \\ \mathrm{H} & -2.307992 & -1.429332 & 1.610128 \\ \mathrm{H} & -3.157449 & -2.456398 & 0.445354 \\ \mathrm{H} & -1.382106 & -2.399871 & 0.451617 \\ \mathrm{H} & -3.593973 & 0.596288 & 0.708233 \\ \mathrm{H} & -4.421199 & -0.490248 & -0.417978 \\ \mathrm{H} & -3.517602 & 0.907617 & -1.035466 \\ \mathrm{~N} & 0.003927 & 1.010862 & 0.011785 \\ \mathrm{H} & 0.895370 & 0.449453 & -0.173012 \\ \mathrm{H} & -0.830983 & 0.356092 & -0.125209 \\ \mathrm{C} & -0.082424 & 2.108628 & -0.989469 \\ \mathrm{C} & 0.016668 & 1.493509 & 1.419889 \\ \mathrm{H} & 0.876143 & 2.144784 & 1.577310 \\ \mathrm{H} & 0.082262 & 0.637037 & 2.089724 \\ \mathrm{H} & -0.898946 & 2.047340 & 1.626264 \\ \mathrm{H} & 0.781081 & 2.766255 & -0.890275 \\ \mathrm{H} & -0.097468 & 1.677807 & -1.990150 \\ \mathrm{H} & -0.994309 & 2.682683 & -0.827285 \\ \mathrm{~N} & 2.422474 & -0.427678 & -0.482373 \\ \mathrm{C} & 2.220005 & -1.883632 & -0.348995 \\ \mathrm{C} & 3.523264 & 0.057863 & 0.374612 \\ \mathrm{H} & 2.676227 & -0.237294 & -1.449209 \\ \mathrm{H} & 1.884501 & -2.112466 & 0.665352 \\ \mathrm{H} & 1.453391 & -2.216207 & -1.051681 \\ \mathrm{H} & 3.136373 & -2.454790 & -0.538863 \\ \mathrm{H} & 3.254763 & -0.079366 & 1.424567 \\ \mathrm{H} & 4.462797 & -0.477157 & 0.192936 \\ \mathrm{H} & 3.690430 & 1.121604 & 0.195759\end{array}$

\section{$\mathrm{H}_{2} \mathrm{SO}_{4} \cdot\left(\left(\mathrm{CH}_{3}\right)_{2} \mathrm{NH}\right)_{2} \cdot\left(\mathrm{CH}_{3}\right)_{2} \mathrm{NH}_{2}{ }^{+}$}

38

$\begin{array}{llcc}\mathrm{S} & 0.000007 & -0.143982 & 0.000031 \\ \mathrm{O} & 0.537025 & -0.999098 & -1.096385 \\ \mathrm{O} & -1.111321 & 0.732080 & -0.526924 \\ \mathrm{O} & -0.537029 & -0.998979 & 1.096531 \\ \mathrm{~N} & -2.910271 & -1.353893 & -0.078223 \\ \mathrm{H} & -2.698968 & -0.421043 & -0.472430 \\ \mathrm{H} & -2.090588 & -1.435763 & 0.585075 \\ \mathrm{C} & -4.210168 & -1.367198 & 0.639101 \\ \mathrm{C} & -2.761858 & -2.377335 & -1.152106 \\ \mathrm{H} & -5.020759 & -1.184267 & -0.065857 \\ \mathrm{H} & -4.348054 & -2.338993 & 1.111548\end{array}$




$\begin{array}{crrr}\mathrm{H} & -4.199204 & -0.589712 & 1.401455 \\ \mathrm{H} & -3.532229 & -2.226451 & -1.907744 \\ \mathrm{H} & -2.862425 & -3.369002 & -0.712711 \\ \mathrm{H} & -1.771300 & -2.259884 & -1.589694 \\ \mathrm{~N} & 2.910444 & -1.353581 & 0.078148 \\ \mathrm{H} & 2.699021 & -0.420697 & 0.472226 \\ \mathrm{H} & 2.090747 & -1.435662 & -0.585094 \\ \mathrm{C} & 2.762206 & -2.376870 & 1.152201 \\ \mathrm{C} & 4.210316 & -1.366829 & -0.639221 \\ \mathrm{H} & 5.020907 & -1.183665 & 0.065675 \\ \mathrm{H} & 4.199218 & -0.589475 & -1.401709 \\ \mathrm{H} & 4.348322 & -2.338686 & -1.111508 \\ \mathrm{H} & 3.532586 & -2.225759 & 1.907785 \\ \mathrm{H} & 1.771651 & -2.259480 & 1.589813 \\ \mathrm{H} & 2.862887 & -3.368595 & 0.712963 \\ \mathrm{O} & 1.111325 & 0.732143 & 0.526889 \\ \mathrm{H} & 0.707642 & 2.417444 & 0.338028 \\ \mathrm{~N} & -0.000152 & 3.112746 & -0.000009 \\ \mathrm{C} & 0.535568 & 3.908100 & -1.133732 \\ \mathrm{C} & -0.536095 & 3.907992 & 1.133684 \\ \mathrm{H} & -0.707764 & 2.417218 & -0.338017 \\ \mathrm{H} & 1.358528 & 4.531748 & -0.785414 \\ \mathrm{H} & 0.889737 & 3.221716 & -1.901098 \\ \mathrm{H} & -0.254224 & 4.538210 & -1.541870 \\ \mathrm{H} & 0.253493 & 4.538405 & 1.541748 \\ \mathrm{H} & -1.359287 & 4.531328 & 0.785356 \\ \mathrm{H} & -0.889999 & 3.221536 & 1.901109\end{array}$

$\left(\mathrm{H}_{2} \mathrm{SO}_{4}\right)_{2} \cdot\left(\left(\mathrm{CH}_{3}\right)_{2} \mathrm{NH}\right)_{2} \cdot\left(\mathrm{CH}_{3}\right)_{2} \mathrm{NH}_{2}^{+}$

45

$\begin{array}{lrrr}\mathrm{S} & 1.884192 & -0.076190 & -1.260217 \\ \mathrm{O} & 0.951264 & -1.204906 & -1.232511 \\ \mathrm{C} & 1.042951 & 3.292035 & 0.938365 \\ \mathrm{H} & 1.802628 & 4.005117 & 0.620381 \\ \mathrm{H} & 0.433800 & 3.723448 & 1.731465 \\ \mathrm{H} & 1.519539 & 2.376014 & 1.279270 \\ \mathrm{~N} & 1.521374 & -1.389638 & 2.123490 \\ \mathrm{H} & 1.998933 & -0.861259 & 1.361622 \\ \mathrm{H} & 0.581763 & -0.940556 & 2.185830 \\ \mathrm{C} & 2.241720 & -1.188110 & 3.410381 \\ \mathrm{C} & 1.373519 & -2.814923 & 1.714848 \\ \mathrm{H} & 2.359753 & -3.273897 & 1.655605 \\ \mathrm{H} & 0.898954 & -2.835042 & 0.736946 \\ \mathrm{H} & 0.761697 & -3.337330 & 2.449023 \\ \mathrm{H} & 3.254439 & -1.579415 & 3.322844 \\ \mathrm{H} & 2.275151 & -0.122545 & 3.630836 \\ \mathrm{H} & 1.709881 & -1.708627 & 4.205766 \\ \mathrm{O} & 2.456970 & 0.217815 & 0.063064 \\ \mathrm{O} & 1.375776 & 1.124501 & -1.930099 \\ \mathrm{~N} & 0.171397 & 2.954684 & -0.222056 \\ \mathrm{C} & -0.471985 & 4.127970 & -0.874171 \\ \mathrm{H} & 0.721272 & 2.408753 & -0.918350 \\ \mathrm{H} & -0.569185 & 2.282482 & 0.077953 \\ \mathrm{H} & -1.104045 & 4.639576 & -0.149497 \\ \mathrm{H} & -1.079966 & 3.777218 & -1.706259 \\ \mathrm{H} & 0.298040 & 4.805860 & -1.240080 \\ \mathrm{C} & -1.796846 & -2.907914 & -2.471055 \\ \mathrm{H} & -1.248354 & -3.583397 & -1.817122\end{array}$




$\begin{array}{cccc}\mathrm{H} & -1.365662 & -2.937018 & -3.470797 \\ \mathrm{H} & -2.845416 & -3.200224 & -2.509169 \\ \mathrm{~N} & -1.689306 & -1.524892 & -1.930431 \\ \mathrm{C} & -2.392506 & -0.488984 & -2.737891 \\ \mathrm{H} & -0.681841 & -1.280328 & -1.831735 \\ \mathrm{H} & -2.037808 & -1.495436 & -0.949050 \\ \mathrm{H} & -2.302412 & 0.460041 & -2.214893 \\ \mathrm{H} & -1.934298 & -0.430175 & -3.724327 \\ \mathrm{H} & -3.443249 & -0.761168 & -2.830956 \\ \mathrm{~S} & -1.882255 & 0.065593 & 1.271585 \\ \mathrm{O} & -3.204123 & 0.595095 & 2.031956 \\ \mathrm{O} & -1.744914 & 1.038112 & 0.182005 \\ \mathrm{O} & -2.167756 & -1.293028 & 0.799337 \\ \mathrm{O} & -0.788564 & 0.116101 & 2.249718 \\ \mathrm{H} & -3.411555 & -0.010532 & 2.758960 \\ \mathrm{O} & 3.079510 & -0.646624 & -2.182800 \\ \mathrm{H} & 3.706275 & 0.069947 & -2.361405\end{array}$

\section{$\left(\mathrm{H}_{2} \mathrm{SO}_{4}\right)_{3} \cdot\left(\left(\mathrm{CH}_{3}\right)_{2} \mathrm{NH}\right)_{2} \cdot\left(\mathrm{CH}_{3}\right)_{2} \mathrm{NH}_{2}{ }^{+}$}

52

$\begin{array}{lccc}\mathrm{S} & -3.223059 & -1.004714 & 0.110782 \\ \mathrm{O} & -3.184802 & 0.459256 & 0.205771 \\ \mathrm{O} & -4.801166 & -1.303294 & 0.076695 \\ \mathrm{O} & -2.678452 & -1.515797 & -1.160938 \\ \mathrm{H} & -4.941985 & -2.256714 & -0.026235 \\ \mathrm{~S} & 1.733615 & 1.848743 & 0.237608 \\ \mathrm{O} & 2.541427 & 1.147581 & 1.237488 \\ \mathrm{O} & 1.922958 & 1.370081 & -1.135131 \\ \mathrm{~S} & 0.670184 & -1.798101 & -1.018165 \\ \mathrm{O} & 0.513655 & -0.639843 & -2.092256 \\ \mathrm{O} & 0.443194 & -1.232439 & 0.293713 \\ \mathrm{O} & 1.937317 & -2.467920 & -1.212688 \\ \mathrm{O} & -0.456124 & -2.747908 & -1.452884 \\ \mathrm{H} & -1.374460 & -2.316114 & -1.301735 \\ \mathrm{O} & -2.664235 & -1.669330 & 1.286508 \\ \mathrm{H} & 1.054684 & 0.144457 & -1.789548 \\ \mathrm{O} & 0.312153 & 1.959279 & 0.617489 \\ \mathrm{O} & 2.304465 & 3.355495 & 0.142584 \\ \mathrm{H} & 2.431112 & 3.700276 & 1.039002 \\ \mathrm{~N} & -0.886784 & 0.055050 & 2.546485 \\ \mathrm{H} & -0.392029 & 0.591122 & 1.816261 \\ \mathrm{H} & -1.456961 & -0.661745 & 2.046037 \\ \mathrm{C} & 0.110598 & -0.629321 & 3.415628 \\ \mathrm{C} & -1.818305 & 0.963734 & 3.273213 \\ \mathrm{H} & -0.418240 & -1.206730 & 4.172718 \\ \mathrm{H} & 0.703921 & -1.288156 & 2.787450 \\ \mathrm{H} & 0.747598 & 0.116849 & 3.888219 \\ \mathrm{H} & -2.345780 & 0.391803 & 4.035072 \\ \mathrm{H} & -1.250054 & 1.769637 & 3.735577 \\ \mathrm{H} & -2.534089 & 1.357628 & 2.555024 \\ \mathrm{~N} & 4.004477 & -1.020943 & 0.108496 \\ \mathrm{H} & 3.290155 & -1.618601 & -0.349857 \\ \mathrm{H} & 3.478811 & -0.256067 & 0.578110 \\ \mathrm{C} & 4.738637 & -1.826186 & 1.126743 \\ \mathrm{C} & 4.874270 & -0.418563 & -0.946955 \\ \mathrm{H} & 5.448816 & -1.184570 & 1.646021\end{array}$




$\begin{array}{lrrr}\mathrm{H} & 4.020165 & -2.235195 & 1.835013 \\ \mathrm{H} & 5.265173 & -2.638573 & 0.628221 \\ \mathrm{H} & 5.621349 & 0.214471 & -0.470459 \\ \mathrm{H} & 5.359321 & -1.216456 & -1.506918 \\ \mathrm{H} & 4.247106 & 0.180727 & -1.603634 \\ \mathrm{~N} & -1.815110 & 2.198579 & -1.349243 \\ \mathrm{H} & -0.913778 & 2.123521 & -0.849334 \\ \mathrm{H} & -2.427250 & 1.498950 & -0.865812 \\ \mathrm{C} & -2.366421 & 3.565607 & -1.129927 \\ \mathrm{C} & -1.664185 & 1.819454 & -2.785110 \\ \mathrm{H} & -3.361711 & 3.621524 & -1.567859 \\ \mathrm{H} & -2.424850 & 3.751588 & -0.059163 \\ \mathrm{H} & -1.710864 & 4.299073 & -1.597573 \\ \mathrm{H} & -2.634771 & 1.903954 & -3.271791 \\ \mathrm{H} & -0.948482 & 2.488817 & -3.260808 \\ \mathrm{H} & -1.313492 & 0.791232 & -2.838144\end{array}$

$\left(\mathrm{H}_{2} \mathrm{SO}_{4}\right)_{4} \cdot\left(\left(\mathrm{CH}_{3}\right)_{2} \mathrm{NH}\right)_{2} \cdot\left(\mathrm{CH}_{3}\right)_{2} \mathrm{NH}_{2}{ }^{+}$

59

$\begin{array}{lccc}\mathrm{S} & -2.372696 & -1.285476 & 0.060031 \\ \mathrm{O} & -2.185594 & -2.771377 & -0.427973 \\ \mathrm{O} & -3.635252 & -1.284841 & 0.781648 \\ \mathrm{O} & -1.190933 & -0.972703 & 0.916234 \\ \mathrm{O} & -2.398900 & -0.402898 & -1.123994 \\ \mathrm{H} & 1.492321 & 3.756597 & -0.035383 \\ \mathrm{~N} & 0.629306 & -2.912814 & 1.984826 \\ \mathrm{H} & -0.085555 & -2.232021 & 1.663919 \\ \mathrm{H} & 1.414193 & -2.787449 & 1.324723 \\ \mathrm{O} & 2.247073 & 0.859336 & -0.717669 \\ \mathrm{O} & 2.016490 & -0.251362 & 1.484939 \\ \mathrm{~S} & 2.956507 & 0.404679 & 0.615788 \\ \mathrm{O} & 3.652354 & 1.581058 & 1.069953 \\ \mathrm{O} & 4.042494 & -0.629489 & 0.148068 \\ \mathrm{H} & 3.583333 & -1.490429 & -0.061316 \\ \mathrm{H} & -4.585655 & 0.113205 & 0.311719 \\ \mathrm{H} & 2.958306 & 3.123306 & 0.414504 \\ \mathrm{~N} & 2.508212 & 3.959059 & -0.001266 \\ \mathrm{C} & 2.735830 & 5.137112 & 0.885331 \\ \mathrm{C} & 3.006404 & 4.126869 & -1.399000 \\ \mathrm{C} & 0.098891 & -4.303130 & 1.843574 \\ \mathrm{C} & 1.054339 & -2.562077 & 3.372484 \\ \mathrm{H} & 1.431414 & -1.543116 & 3.368327 \\ \mathrm{H} & 1.836243 & -3.251765 & 3.687524 \\ \mathrm{H} & 0.194494 & -2.650942 & 4.034944 \\ \mathrm{H} & -0.799132 & -4.402287 & 2.451031 \\ \mathrm{H} & 0.860127 & -5.004424 & 2.182177 \\ \mathrm{H} & -0.130198 & -4.479212 & 0.795979 \\ \mathrm{H} & 2.489823 & 4.966397 & -1.861512 \\ \mathrm{H} & 4.079161 & 4.312071 & -1.373801 \\ \mathrm{H} & 2.798833 & 3.208925 & -1.944485 \\ \mathrm{H} & 3.804552 & 5.336281 & 0.947483 \\ \mathrm{H} & 2.216562 & 5.999887 & 0.471171 \\ \mathrm{H} & 2.343148 & 4.909425 & 1.874773 \\ \mathrm{~S} & -1.079870 & 2.430002 & -0.063517 \\ \mathrm{O} & -0.301193 & 3.637355 & -0.148496 \\ \mathrm{O} & -0.816621 & 1.619479 & -1.373696 \\ \mathrm{O} & -2.502059 & 2.546426 & 0.166245 \\ \mathrm{H} & -1.389444 & 0.783774 & -1.383123\end{array}$




$\begin{array}{lccc}\mathrm{O} & -0.420307 & 1.579097 & 1.078303 \\ \mathrm{H} & -0.709270 & 0.617151 & 1.061970 \\ \mathrm{~N} & -4.945722 & 1.035926 & -0.015821 \\ \mathrm{C} & -5.891295 & 1.595198 & 0.991879 \\ \mathrm{C} & -5.514579 & 0.873137 & -1.386178 \\ \mathrm{H} & -4.104955 & 1.632948 & -0.062688 \\ \mathrm{H} & -6.747111 & 0.928402 & 1.085450 \\ \mathrm{H} & -5.375840 & 1.672442 & 1.947602 \\ \mathrm{H} & -6.220502 & 2.581502 & 0.668113 \\ \mathrm{H} & -6.371137 & 0.202551 & -1.335869 \\ \mathrm{H} & -5.823999 & 1.846445 & -1.764557 \\ \mathrm{H} & -4.742356 & 0.444325 & -2.021292 \\ \mathrm{~S} & 1.469992 & -2.570532 & -1.523705 \\ \mathrm{O} & 2.366907 & -2.667208 & -0.356305 \\ \mathrm{O} & 0.233173 & -3.332942 & -1.358936 \\ \mathrm{H} & -1.317764 & -2.887298 & -0.902975 \\ \mathrm{O} & 1.243539 & -1.189585 & -1.963017 \\ \mathrm{H} & 1.811543 & 0.087194 & -1.203172 \\ \mathrm{O} & 2.326650 & -3.305445 & -2.663233 \\ \mathrm{H} & 1.803267 & -3.349708 & -3.478058\end{array}$

$\left(\mathrm{H}_{2} \mathrm{SO}_{4}\right)_{2} \cdot\left(\left(\mathrm{CH}_{3}\right)_{2} \mathrm{NH}\right)_{3} \cdot\left(\mathrm{CH}_{3}\right)_{2} \mathrm{NH}_{2}{ }^{+}$

55

$\begin{array}{lrrr}\mathrm{S} & -1.420686 & -0.013292 & 0.876741 \\ \mathrm{O} & -2.709473 & -0.019954 & 1.622558 \\ \mathrm{O} & -0.613277 & -1.226876 & 1.198038 \\ \mathrm{O} & -0.631687 & 1.205635 & 1.219415 \\ \mathrm{O} & -1.722794 & -0.027408 & -0.613129 \\ \mathrm{H} & 0.762404 & -0.890500 & 2.032087 \\ \mathrm{~N} & -0.012287 & -1.955128 & -1.674721 \\ \mathrm{H} & 0.900100 & -1.794940 & -1.203061 \\ \mathrm{H} & -0.653456 & -1.205734 & -1.314184 \\ \mathrm{O} & 3.056335 & 0.705923 & 0.449505 \\ \mathrm{O} & 2.003152 & 0.599017 & -1.773206 \\ \mathrm{~S} & 2.881095 & -0.051192 & -0.797279 \\ \mathrm{O} & 2.525993 & -1.457107 & -0.553097 \\ \mathrm{H} & 2.189610 & -0.063184 & 1.855599 \\ \mathrm{~N} & 1.653150 & -0.628977 & 2.538170 \\ \mathrm{H} & 0.062799 & 2.123473 & 0.091041 \\ \mathrm{~N} & 0.540122 & 2.685093 & -0.670347 \\ \mathrm{H} & 1.115490 & 1.985035 & -1.179022 \\ \mathrm{C} & 1.440882 & 3.700433 & -0.063190 \\ \mathrm{C} & -0.501747 & 3.232594 & -1.574647 \\ \mathrm{C} & -0.544769 & -3.279735 & -1.256581 \\ \mathrm{C} & 1.298528 & 0.199942 & 3.722023 \\ \mathrm{H} & 0.167495 & -4.058038 & -1.528967 \\ \mathrm{H} & -0.693343 & -3.250422 & -0.179553 \\ \mathrm{H} & -1.493828 & -3.459642 & -1.761676 \\ \mathrm{H} & -1.129263 & 2.404990 & -1.901256 \\ \mathrm{H} & -1.105081 & 3.956792 & -1.027612 \\ \mathrm{H} & -0.032273 & 3.716062 & -2.431186 \\ \mathrm{H} & 2.195309 & 3.175486 & 0.519534 \\ \mathrm{H} & 1.924132 & 4.277311 & -0.851707 \\ \mathrm{H} & 0.858509 & 4.362249 & 0.577109 \\ \mathrm{H} & 0.687817 & 1.031624 & 3.376927 \\ \mathrm{H} & 2.208172 & 0.557642 & 4.204208 \\ \mathrm{H} & 0.723889 & -0.407892 & 4.420120 \\ \mathrm{C} & 0.183287 & -1.800931 & -3.141432\end{array}$




$\begin{array}{rrrr}\mathrm{H} & 0.630255 & -0.826161 & -3.323640 \\ \mathrm{H} & -0.779519 & -1.884304 & -3.645132 \\ \mathrm{H} & 0.854527 & -2.581397 & -3.498353 \\ \mathrm{C} & 2.426408 & -1.859975 & 2.849816 \\ \mathrm{H} & 1.824229 & -2.510100 & 3.483564 \\ \mathrm{H} & 3.347089 & -1.590577 & 3.367342 \\ \mathrm{H} & 2.660882 & -2.358630 & 1.911321 \\ \mathrm{~N} & -4.529793 & 0.031449 & -0.199802 \\ \mathrm{H} & -3.771803 & 0.038987 & -0.896740 \\ \mathrm{H} & -3.915982 & 0.011022 & 0.692317 \\ \mathrm{C} & -5.330470 & -1.213802 & -0.307014 \\ \mathrm{C} & -5.317748 & 1.287855 & -0.262270 \\ \mathrm{H} & -5.845948 & 1.353721 & -1.213779 \\ \mathrm{H} & -6.035267 & 1.297128 & 0.557343 \\ \mathrm{H} & -4.637460 & 2.130918 & -0.153744 \\ \mathrm{H} & -6.048715 & -1.244990 & 0.511403 \\ \mathrm{H} & -5.858821 & -1.240688 & -1.260373 \\ \mathrm{H} & -4.658669 & -2.066864 & -0.227375 \\ \mathrm{O} & 4.306037 & -0.039489 & -1.557888 \\ \mathrm{H} & 4.981307 & -0.399902 & -0.964846\end{array}$

$\left(\mathrm{H}_{2} \mathrm{SO}_{4}\right)_{3} \cdot\left(\left(\mathrm{CH}_{3}\right)_{2} \mathrm{NH}\right)_{3} \cdot\left(\mathrm{CH}_{3}\right)_{2} \mathrm{NH}_{2}{ }^{+}$

62

$\begin{array}{lrrr}\text { S } & 3.032820 & 0.661807 & -0.113686 \\ \mathrm{O} & 3.350035 & -0.599533 & 0.580378 \\ \mathrm{O} & 4.356585 & 1.597309 & -0.016618 \\ \mathrm{O} & 2.778380 & 0.450623 & -1.541293 \\ \mathrm{H} & 5.070967 & 1.179799 & -0.519015 \\ \mathrm{~S} & -0.764419 & -1.978540 & 0.085433 \\ \mathrm{O} & -2.090310 & -2.547296 & -0.120862 \\ \mathrm{O} & -0.410280 & -1.069218 & -1.184814 \\ \mathrm{O} & -0.739211 & -1.064805 & 1.266173 \\ \mathrm{H} & -1.080503 & -0.321581 & -1.262381 \\ \mathrm{~S} & -2.445001 & 1.775477 & -0.151720 \\ \mathrm{O} & -2.203531 & 3.135340 & -0.612472 \\ \mathrm{O} & -2.243233 & 0.738261 & -1.192134 \\ \mathrm{O} & -3.705260 & 1.554721 & 0.555155 \\ \mathrm{O} & -1.238667 & 1.568602 & 0.909117 \\ \mathrm{H} & -1.112691 & 0.606595 & 1.097712 \\ \mathrm{O} & 2.039604 & 1.468566 & 0.588843 \\ \mathrm{O} & 0.347191 & -2.928286 & 0.107782 \\ \mathrm{~N} & 0.518059 & 3.644141 & -0.374281 \\ \mathrm{H} & 0.934756 & 2.752785 & -0.046644 \\ \mathrm{H} & -0.492492 & 3.458415 & -0.559682 \\ \mathrm{C} & 0.605447 & 4.635794 & 0.733455 \\ \mathrm{C} & 1.202637 & 4.055822 & -1.630191 \\ \mathrm{H} & 0.155300 & 5.573652 & 0.411026 \\ \mathrm{H} & 0.057399 & 4.239042 & 1.585585 \\ \mathrm{H} & 1.651158 & 4.789001 & 0.996320 \\ \mathrm{H} & 0.736379 & 4.961904 & -2.014715 \\ \mathrm{H} & 2.256450 & 4.234520 & -1.420809 \\ \mathrm{H} & 1.108305 & 3.250583 & -2.356680 \\ \mathrm{~N} & 1.642328 & -1.185807 & 2.685752 \\ \mathrm{H} & 2.337459 & -1.006967 & 1.929555 \\ \mathrm{H} & 0.716411 & -1.141387 & 2.207686 \\ \mathrm{C} & 1.832990 & -2.556222 & 3.235516 \\ \mathrm{C} & 1.740549 & -0.087322 & 3.686113 \\ \mathrm{H} & 1.100792 & -2.731037 & 4.023079 \\ & & & \\ & & & \end{array}$




$\begin{array}{llll}\mathrm{H} & 1.678887 & -3.269550 & 2.428034 \\ \mathrm{H} & 2.840401 & -2.646895 & 3.640139 \\ \mathrm{H} & 0.958536 & -0.208864 & 4.434737 \\ \mathrm{H} & 2.719687 & -0.122702 & 4.162473 \\ \mathrm{H} & 1.620982 & 0.857843 & 3.160161 \\ \mathrm{~N} & 2.182803 & -2.283436 & -1.977017 \\ \mathrm{H} & 2.331657 & -1.258348 & -1.876236 \\ \mathrm{H} & 1.528477 & -2.544016 & -1.213189 \\ \mathrm{C} & 1.533165 & -2.568134 & -3.287448 \\ \mathrm{C} & 3.483907 & -2.978730 & -1.771473 \\ \mathrm{H} & 1.350114 & -3.638829 & -3.369332 \\ \mathrm{H} & 0.591179 & -2.026803 & -3.322048 \\ \mathrm{H} & 2.191271 & -2.240575 & -4.091281 \\ \mathrm{H} & 3.322337 & -4.055814 & -1.789181 \\ \mathrm{H} & 4.169533 & -2.693950 & -2.568680 \\ \mathrm{H} & 3.884684 & -2.666733 & -0.809632 \\ \mathrm{~N} & -4.495960 & -1.051692 & 0.004978 \\ \mathrm{H} & -4.240744 & -0.072693 & 0.271889 \\ \mathrm{H} & -3.604313 & -1.584349 & -0.026284 \\ \mathrm{C} & -5.383545 & -1.641050 & 1.044142 \\ \mathrm{C} & -5.080583 & -1.018465 & -1.366048 \\ \mathrm{H} & -5.617505 & -2.670883 & 0.777459 \\ \mathrm{H} & -4.864209 & -1.617985 & 2.000806 \\ \mathrm{H} & -6.299210 & -1.054796 & 1.108664 \\ \mathrm{H} & -5.287338 & -2.036346 & -1.694160 \\ \mathrm{H} & -6.000609 & -0.435824 & -1.345754 \\ \mathrm{H} & -4.356344 & -0.543994 & -2.024526\end{array}$

$\left(\mathrm{H}_{2} \mathrm{SO}_{4}\right)_{4} \cdot\left(\left(\mathrm{CH}_{3}\right)_{2} \mathrm{NH}\right)_{3} \cdot\left(\mathrm{CH}_{3}\right)_{2} \mathrm{NH}_{2}{ }^{+}$

69

$\begin{array}{lccc}\mathrm{S} & -2.141725 & -2.245959 & -0.340778 \\ \mathrm{O} & -3.383788 & -2.867586 & 0.107556 \\ \mathrm{O} & -2.421891 & -1.085948 & -1.228578 \\ \mathrm{O} & -1.448698 & -3.358688 & -1.236861 \\ \mathrm{H} & -4.664152 & -1.764375 & -0.042790 \\ \mathrm{H} & -0.493020 & -3.147997 & -1.349122 \\ \mathrm{~S} & -2.562379 & 1.881271 & 0.383805 \\ \mathrm{O} & -1.803345 & 1.310776 & -0.850066 \\ \mathrm{O} & -2.373041 & 3.314240 & 0.335027 \\ \mathrm{O} & -1.835470 & 1.266983 & 1.619522 \\ \mathrm{H} & -1.988940 & 0.298616 & -0.983606 \\ \mathrm{~N} & -5.350996 & -0.981846 & -0.168754 \\ \mathrm{C} & -6.445779 & -1.104632 & 0.831817 \\ \mathrm{H} & -6.981003 & -2.038186 & 0.663686 \\ \mathrm{H} & -7.126621 & -0.260424 & 0.729913 \\ \mathrm{H} & -6.007551 & -1.105437 & 1.828439 \\ \mathrm{C} & -5.798709 & -0.970895 & -1.589956 \\ \mathrm{H} & -6.325205 & -1.900060 & -1.803627 \\ \mathrm{H} & -4.913247 & -0.893946 & -2.217610 \\ \mathrm{H} & -6.458607 & -0.119893 & -1.754712 \\ \mathrm{H} & -0.831316 & 1.220081 & 1.501994 \\ \mathrm{H} & -4.812656 & -0.119327 & 0.019866 \\ \mathrm{O} & -1.207646 & -1.888471 & 0.728104 \\ \mathrm{O} & -3.923800 & 1.403356 & 0.439370 \\ \mathrm{~S} & 1.785346 & 1.855096 & 0.864498 \\ \mathrm{O} & 3.114036 & 1.445819 & 1.322437 \\ \mathrm{O} & 1.769266 & 1.713229 & -0.745922 \\ \mathrm{O} & 1.502937 & 3.273227 & 1.056230\end{array}$




$\begin{array}{lrrr}\mathrm{H} & 4.358713 & 0.717607 & 0.480272 \\ \mathrm{H} & 0.563938 & 4.180188 & -0.009607 \\ \mathrm{~S} & 2.358737 & -2.086063 & -1.268290 \\ \mathrm{O} & 3.116703 & -2.000777 & -0.000554 \\ \mathrm{O} & 1.254685 & -3.034724 & -1.199577 \\ \mathrm{O} & 3.474773 & -2.726242 & -2.255678 \\ \mathrm{H} & 1.737516 & 0.767232 & -1.028586 \\ \mathrm{~N} & 5.025215 & 0.045272 & 0.032233 \\ \mathrm{C} & 6.210701 & -0.141448 & 0.911011 \\ \mathrm{H} & 6.750780 & 0.801158 & 0.990202 \\ \mathrm{H} & 6.860233 & -0.907027 & 0.488117 \\ \mathrm{H} & 5.868769 & -0.447957 & 1.898162 \\ \mathrm{C} & 5.340434 & 0.495022 & -1.352855 \\ \mathrm{H} & 5.867123 & 1.447393 & -1.307527 \\ \mathrm{H} & 4.403931 & 0.610582 & -1.894471 \\ \mathrm{H} & 5.960713 & -0.254246 & -1.842992 \\ \mathrm{H} & 3.050477 & -2.954993 & -3.096185 \\ \mathrm{H} & 4.468463 & -0.831551 & -0.010084 \\ \mathrm{O} & 0.735077 & 0.951233 & 1.370078 \\ \mathrm{O} & 2.018559 & -0.776492 & -1.818267 \\ \mathrm{~N} & -0.143535 & 4.687005 & -0.591057 \\ \mathrm{C} & 0.175962 & 4.503205 & -2.033814 \\ \mathrm{C} & -0.228890 & 6.103236 & -0.141027 \\ \mathrm{H} & -1.034629 & 4.205863 & -0.367516 \\ \mathrm{H} & 1.132417 & 4.977608 & -2.249831 \\ \mathrm{H} & 0.241933 & 3.435889 & -2.234048 \\ \mathrm{H} & -0.609664 & 4.956002 & -2.637684 \\ \mathrm{H} & 0.732952 & 6.589218 & -0.299533 \\ \mathrm{H} & -1.004377 & 6.617364 & -0.707574 \\ \mathrm{H} & -0.476851 & 6.114907 & 0.918932 \\ \mathrm{~N} & 1.098102 & -2.570562 & 1.982161 \\ \mathrm{H} & 1.900575 & -2.323166 & 1.380029 \\ \mathrm{H} & 0.245055 & -2.283668 & 1.452246 \\ \mathrm{C} & 1.057881 & -4.051154 & 2.143149 \\ \mathrm{C} & 1.155749 & -1.797895 & 3.255481 \\ \mathrm{H} & 0.164997 & -4.321995 & 2.704514 \\ \mathrm{H} & 1.023014 & -4.497716 & 1.151374 \\ \mathrm{H} & 1.949524 & -4.383037 & 2.673800 \\ \mathrm{H} & 0.263139 & -2.018117 & 3.839395 \\ \mathrm{H} & 2.045057 & -2.088755 & 3.814263 \\ \mathrm{H} & 1.186406 & -0.738559 & 3.006682 \\ & & & \\ & & & \end{array}$

$\left(\mathrm{H}_{2} \mathrm{SO}_{4}\right)_{4} \cdot\left(\left(\mathrm{CH}_{3}\right)_{2} \mathrm{NH}\right)_{4} \cdot\left(\mathrm{CH}_{3}\right)_{2} \mathrm{NH}_{2}{ }^{+}$

79

$\begin{array}{lccc}\mathrm{S} & -2.966122 & 2.112272 & -0.359731 \\ \mathrm{O} & -2.503870 & 3.382677 & -0.895659 \\ \mathrm{O} & -4.247648 & 2.184809 & 0.353956 \\ \mathrm{O} & -1.830439 & 1.757904 & 0.728873 \\ \mathrm{H} & 0.614595 & 3.996402 & 0.272550 \\ \mathrm{H} & -1.921750 & 0.815791 & 1.011242 \\ \mathrm{~S} & -2.426808 & -1.896367 & 0.276929 \\ \mathrm{O} & -1.835325 & -1.313952 & -1.119545 \\ \mathrm{O} & -1.656411 & -3.135359 & 0.440385 \\ \mathrm{O} & -3.857105 & -2.104239 & 0.074076 \\ \mathrm{H} & -2.205439 & -0.388876 & -1.274886 \\ \mathrm{~N} & -0.238765 & 4.574631 & 0.130447 \\ \mathrm{C} & 0.105937 & 5.699856 & -0.779759 \\ \mathrm{H} & 0.887330 & 6.304114 & -0.321351\end{array}$




\begin{tabular}{|c|c|c|c|}
\hline $\mathrm{H}$ & -0.783991 & 6.301511 & -0.959359 \\
\hline $\mathrm{H}$ & 0.467800 & 5.286595 & -1.719664 \\
\hline $\mathrm{C}$ & -0.740428 & 4.997076 & 1.466881 \\
\hline $\mathrm{H}$ & 0.038890 & 5.558979 & 1.979722 \\
\hline $\mathrm{H}$ & -0.991510 & 4.101336 & 2.031061 \\
\hline $\mathrm{H}$ & -1.630585 & 5.610719 & 1.336372 \\
\hline $\mathrm{H}$ & -0.975622 & 3.991782 & -0.315966 \\
\hline $\mathrm{O}$ & -2.974243 & 0.998227 & -1.334394 \\
\hline $\mathrm{O}$ & -2.114616 & -0.891373 & 1.303442 \\
\hline S & 2.656375 & -1.819836 & -0.160125 \\
\hline $\mathrm{O}$ & 4.105865 & -1.907055 & -0.011124 \\
\hline $\mathrm{O}$ & 2.080114 & -1.183517 & 1.217292 \\
\hline $\mathrm{O}$ & 1.970879 & -3.116605 & -0.211321 \\
\hline $\mathrm{H}$ & 5.122429 & -0.489301 & -0.240592 \\
\hline $\mathrm{H}$ & -0.483514 & -3.053493 & 1.725607 \\
\hline$S$ & 2.752421 & 2.236415 & 0.192779 \\
\hline $\mathrm{O}$ & 3.918574 & 2.383102 & -0.687052 \\
\hline $\mathrm{O}$ & 3.013956 & 1.220164 & 1.239394 \\
\hline $\mathrm{O}$ & 1.540730 & 1.669483 & -0.699781 \\
\hline $\mathrm{H}$ & 2.390316 & -0.224403 & 1.299182 \\
\hline $\mathrm{N}$ & 5.709809 & 0.365435 & -0.324278 \\
\hline $\mathrm{C}$ & 6.648565 & 0.211866 & -1.466532 \\
\hline $\mathrm{H}$ & 7.315740 & -0.628854 & -1.279005 \\
\hline $\mathrm{H}$ & 7.227368 & 1.127413 & -1.582333 \\
\hline $\mathrm{H}$ & 6.070302 & 0.027918 & -2.370648 \\
\hline $\mathrm{C}$ & 6.363143 & 0.627672 & 0.987745 \\
\hline $\mathrm{H}$ & 7.003687 & -0.213981 & 1.249150 \\
\hline $\mathrm{H}$ & 5.579089 & 0.755603 & 1.731207 \\
\hline $\mathrm{H}$ & 6.953973 & 1.540040 & 0.915321 \\
\hline $\mathrm{H}$ & 1.753993 & 0.751619 & -0.993716 \\
\hline $\mathrm{H}$ & 5.040319 & 1.152725 & -0.517868 \\
\hline $\mathrm{O}$ & 2.233810 & -0.911011 & -1.237680 \\
\hline $\mathrm{O}$ & 2.216253 & 3.490972 & 0.697725 \\
\hline $\mathrm{N}$ & 0.235961 & -2.934725 & 2.472403 \\
\hline $\mathrm{C}$ & 0.942491 & -4.228194 & 2.693227 \\
\hline $\mathrm{C}$ & -0.414178 & -2.368644 & 3.687996 \\
\hline $\mathrm{H}$ & 0.907308 & -2.248055 & 2.085928 \\
\hline $\mathrm{H}$ & 0.218242 & -4.980332 & 3.003731 \\
\hline $\mathrm{H}$ & 1.421389 & -4.514859 & 1.759809 \\
\hline $\mathrm{H}$ & 1.695716 & -4.095858 & 3.469205 \\
\hline $\mathrm{H}$ & -1.118329 & -3.099025 & 4.084859 \\
\hline $\mathrm{H}$ & 0.349044 & -2.146517 & 4.433007 \\
\hline $\mathrm{H}$ & -0.947814 & -1.466659 & 3.397532 \\
\hline $\mathrm{N}$ & 0.054246 & -3.125698 & -2.245006 \\
\hline $\mathrm{H}$ & 0.785032 & -3.173506 & -1.501917 \\
\hline $\mathrm{H}$ & -0.626174 & -2.425327 & -1.902331 \\
\hline $\mathrm{C}$ & 0.674611 & -2.639678 & -3.510129 \\
\hline $\mathrm{C}$ & -0.626773 & -4.446652 & -2.359578 \\
\hline $\mathrm{H}$ & -0.103403 & -2.496564 & -4.259133 \\
\hline $\mathrm{H}$ & 1.186014 & -1.703856 & -3.298133 \\
\hline $\mathrm{H}$ & 1.392856 & -3.380870 & -3.858767 \\
\hline $\mathrm{H}$ & -1.395715 & -4.385618 & -3.128924 \\
\hline $\mathrm{H}$ & 0.108934 & -5.203135 & -2.629914 \\
\hline $\mathrm{H}$ & -1.082771 & -4.676305 & -1.399476 \\
\hline $\mathrm{N}$ & -5.748764 & -0.042848 & -0.049042 \\
\hline $\mathrm{C}$ & -6.227287 & 0.041237 & -1.456604 \\
\hline $\mathrm{C}$ & -6.822797 & -0.216439 & 0.963689 \\
\hline $\mathrm{H}$ & -6.367133 & -0.264018 & 1.951503 \\
\hline $\mathrm{H}$ & -7.365776 & -1.139732 & 0.764263 \\
\hline $\mathrm{H}$ & -7.503477 & 0.632733 & 0.915399 \\
\hline
\end{tabular}




$\begin{array}{lrrr}\text { H } & -5.199403 & 0.829988 & 0.159425 \\ \mathrm{H} & -5.067212 & -0.824479 & 0.027072 \\ \mathrm{H} & -5.359680 & 0.196109 & -2.094655 \\ \mathrm{H} & -6.737901 & -0.883634 & -1.722881 \\ \mathrm{H} & -6.908355 & 0.886152 & -1.551244\end{array}$

\section{References}

Becke, A.D., J. Chem. Phys. 98, 5648 (1993).

Frisch, M.J., et al. Gaussian 09, Revision A.01, (2009).

Lide, D., ed.: CRC Handbook of Chemistry and Physics, 91th Edition, CRC Press/Taylor and Francis, Boca Montgomery Jr, J. A., et al., J. Chem. Phys. 110, 2822 (1999).

Nadykto, A.B., and Yu, F., J. Geophys. Res. 108, 4717 (2003).

Sedo, G., et al. J. Mol. Spectrosc. 251, 4 (2008). 